\title{
Cosmic evolution of lepton flavor charges
}

\author{
Chee Sheng Fong $\odot^{*}$ \\ Centro de Ciências Naturais e Humanas, Universidade Federal do ABC, \\ 09.210-170 Santo André, São Paulo, Brazil
}

(Received 17 September 2021; accepted 16 January 2022; published 8 February 2022)

\begin{abstract}
In the early Universe above the weak scale, both baryon $B$ and lepton $L$ numbers are violated by nonperturabive effects in the Standard Model while $B-L$ remains conserved. Introducing new physics which violates perturbatively $L$ and/or $B$, one can generate dynamically a nonzero $B-L$ charge and hence a nonzero $B$ charge. In this work, we focus on the former scenario, which is also known as leptogenesis. We show how to describe the evolutions of lepton flavor charges taking into account the complete Standard Model lepton flavor and spectator effects in a unified and lepton flavor basis-independent way. The recipe we develop can be applied to any leptogenesis model with arbitrary number of new scalars carrying nonzero hypercharges and is valid for cosmic temperature ranging from $10^{15} \mathrm{GeV}$ down to the weak scale. We demonstrate that in order to describe the physics in a basis-independent manner and to include lepton flavor effect consistently it is necessary to describe both left-handed and right-handed lepton charges in terms of density matrices. This is a crucial point since physics should be basis independent. As examples, we apply the formalism to type-I and type-II leptogenesis models where in the latter case a flavor-covariant formalism is indispensable.
\end{abstract}

DOI: 10.1103/PhysRevD.105.043004

\section{INTRODUCTION}

In the early Universe, if the cosmic temperature is above the weak scale, the thermal bath contains all the degrees of freedom of the Standard Model (SM) and perhaps other new physics degrees of freedom as well if they are kinematically accessible. To generate a cosmic baryon asymmetry dynamically (baryogenesis), one needs to violate at least the baryon number $B$ of the SM. Above the weak scale when the SM $B$-violating process is in thermal equilibrium [1], one needs to identify other charges which are not in thermal equilibrium such that the charge is effectively conserved and can remain nonzero. In the SM, one identifies the baryon minus lepton number $B-L$ as the exactly conserved charge. If one introduces new physics which perturbatively violates $B-L$, together with violation of charge $C$ and charge parity $C P$, a nonzero $B-L$ charge can be dynamically generated. Since $B$ is not orthogonal to $B-L$, we have

$$
B=c(B-L),
$$

\footnotetext{
*sheng.fong@ufabc.edu.br
}

Published by the American Physical Society under the terms of the Creative Commons Attribution 4.0 International license. Further distribution of this work must maintain attribution to the author(s) and the published article's title, journal citation, and DOI. Funded by SCOAP ${ }^{3}$. with $c \neq 0$, implying a nonzero $B$ is generated as well. After baryogenesis is completed, i.e., $(B-L)$-violating interaction goes out of equilibrium, while $B-L$ charge remains conserved, it is important to note that, since $B$ is not a conserved charge, it can (and in general will) evolve with cosmic temperature. In other words, the coefficient $c$ that relates $B$ and $B-L$ in Eq. (1) is temperature dependent since it depends on the effective charges of the thermal bath. How can effective charges arise in the early Universe? They arise as the cosmic temperature increases when some of the SM interactions go out of equilibrium. In principle, baryogenesis does not have to go through $B-L$ but can proceed through other effective charges $\mathcal{Q}$ which are not completely orthogonal to $B$ [2],

$$
B=\sum_{\mathcal{Q}} c_{\mathcal{Q}} \mathcal{Q}
$$

with $c_{\mathcal{Q}} \neq 0$. In Ref. [3], we have classified all effective charges of the SM and its minimal supersymmetric extension, 16 in the former and 18 in the latter, and this opens up a new avenue for baryogenesis.

In this work, we focus on baryogenesis scenario through the violation of $B-L$, which can come from perturbative interaction which violates $L$ and/or $B$. We consider the former scenario, which is also known as leptogenesis [4]. First of all, we show that in order describe leptogenesis in a basis-independent manner one needs to describe both the number asymmetries in lepton doublet $\ell$ and $\operatorname{singlet} E$ in 
term of matrices of number densities in their respective flavor spaces (we will denote them densities matrices) [5]. It is of fundamental importance since physics should not depend on a particular basis. While the computation of leptogenesis is usually carried out in a charged lepton mass basis, one should be cautious that this description has limited validity, and in particular, if the result is basis dependent, then it is a red flag that something must be wrong. In this flavor-covariant formalism [6,7], the SM lepton flavor effect is consistently taken into account. ${ }^{1}$ With the effective charges identified in Ref. [3], we are able to include the complete spectator effects due to quark Yukawa and SM sphaleron interactions in a unified manner, which to our knowledge has not been carried out before. (See Ref. [9], in which the spectator effects related to tau and bottom-quark Yukawa interactions are investigated.) In Ref. [10], asymmetry in $E$ is not taken in account, and as a result, one cannot obtain a fully basis-independent result. In Refs. [6,7], asymmetry in singlet $E$ is considered while other spectator effects $[11,12]$ pertaining to quark Yukawa and SM sphaleron interactions are not considered.

This article is organized as follows. In Sec. II, we review the effective symmetries and charges of the SM in the early Universe. In Sec. III, we write down the flavor-covariant Boltzmann equations, taking into account the complete lepton flavor and spectator effects due to quark Yukawa and the SM sphaleron interactions. These results are completely general and, together with the equations in Appendix C, can be applied to any leptogenesis model (with arbitrary number of new scalars carrying nonzero hypercharges) for cosmic temperature ranging from $10^{15} \mathrm{GeV}$ down to the weak scale. In Sec. IV, we apply our results to type-I and type-II leptogenesis models. Finally, we conclude in Sec. V. In Appendix A, we discuss how number density asymmetry matrices are related to matrices of chemical potentials; in Appendix B, we show how the flavor-covariant structure can be derived using Sigl-Raffelt formalism [5]; and in Appendix D, we discuss how to determine the transition temperatures related to spectator effects.

\section{EFFECTIVE SYMMETRIES AND CHARGES}

In the early Universe, due to the additional scale related to cosmic expansion, one should consider effective symmetries and charges. To illustrate this point, let us consider the early Universe which is dominated by radiation energy density $\rho_{r} \propto T^{4}$ with temperature $T$ and is expanding with the Hubble rate $\mathcal{H} \propto \sqrt{\rho_{r}} / M_{\mathrm{Pl}} \propto T^{2} / M_{\mathrm{pl}}$, where $M_{\mathrm{Pl}}=$ $1.22 \times 10^{19} \mathrm{GeV}$ is the Planck scale. Taking all particles to be massless, the interaction rates among the particles have to scale as $T$. At sufficiently high $T$, all of those interactions

\footnotetext{
${ }^{1}$ Reference [8] develops a flavor-covariant formalism which takes into account the flavors of left-handed SM leptons as well as the massive right-handed neutrinos in the type-I seesaw model.
}

will be slower than the Hubble rate. In this case, if one assigns a quantum number or charge to each type of particle, the charge will be effectively conserved since all particle-number-changing processes are out of thermal equilibrium (effectively do not occur within a Hubble time). In the SM, with three families $\alpha=1,2,3$ of quark $Q_{\alpha}$ and lepton $\ell_{\alpha}$ doublets, charged lepton $E_{\alpha}$, up-type $U_{\alpha}$ and down-type $D_{\alpha}$ quarks singlets, and a Higgs doublet, one will expect to have up to 16 effective charges or the associated global $U(1)$ symmetries. One can conveniently choose linear combinations of $U(1)$ charges which are subsequently broken as the cosmic temperature decreases. This choice leads to $U(1)_{x}$ with [3] $]^{2}$

$$
\begin{aligned}
x= & \left\{t, u, B, \tau, u-b, u-c, \mu, B_{3}-B_{2}, u-s, B_{3}\right. \\
& \left.+B_{2}-2 B_{1}, u-d, e, B / 3-L_{\alpha}, Y\right\},
\end{aligned}
$$

where we have denoted the charge associated to each type of particle as $\left\{U_{1}, U_{2}, U_{3}\right\}=\{u, c, t\},\left\{D_{1}, D_{2}, D_{3}\right\}=$ $\{d, s, b\}$, and $\left\{E_{1}, E_{2}, E_{3}\right\}=\{e, \mu, \tau\}$ and $B_{\alpha}$ refers to baryon flavor number with the total baryon number $B=B_{1}+B_{2}+B_{3}$, while $L_{\alpha}$ refers to lepton flavor number with the total lepton number $L=L_{1}+L_{2}+L_{3}$. Out of these $16 U(1)_{x}$, only the last four remain exact before the electroweak (EW) symmetry breaking: hypercharge gauge symmetry $Y$ and the three $B / 3-L_{a}$ accidental (global) symmetries. The rest of the effective symmetries are broken by the Yukawa and nonperturbative sphaleron interactions.

In the absence of neutrino mass, the SM Lagrangian contains four accidental $U(1)$ symmetries: the total baryon number $U(1)_{B}$ and three lepton flavors $U(1)_{L_{\alpha}}$. Nevertheless, there are fewer actual accidental symmetries of the SM due to the Adler-Bell-Jackiw anomaly. We can determine if any of the accidental symmetry $U(1)_{x}$ is preserved from its anomaly coefficient associated with the triangle diagram $U(1)_{x}-S U(N)-S U(N)$,

$$
A_{x N N} \equiv \sum_{i} c\left(R_{i}\right) g_{i} q_{i}^{x}
$$

where the sum is over all fermions $i$ of degeneracy $g_{i}$, charge $q_{i}^{x}$ under $U(1)_{x}$, and representation $R_{i}$ under $S U(N \geq 2)$ gauge group with $c\left(R_{i}\right)=\frac{1}{2}$ in the fundamental representation and $c\left(R_{i}\right)=N$ in the adjoint representation. Since the contribution of each fermion $i$ to the $S U(N)$ sphaleron-induced effective operator is proportional to $c\left(R_{i}\right)$, the effective operator is given by [3]

$$
\mathcal{O}_{\mathrm{SU}(\mathrm{N})} \sim \prod_{i} \Psi_{i}^{2 g_{i} c\left(R_{i}\right)} .
$$

In the SM, we see that $U(1)_{B}$ and $U(1)_{L_{\alpha}}$ are anomalous [13] with anomaly coefficients

\footnotetext{
${ }^{2}$ In the minimal supersymmetric SM, there are 18 effective symmetries and corresponding charges.
} 


$$
\begin{gathered}
A_{B 22} \equiv \frac{1}{2} \times 3\left(3 \times \frac{1}{3}\right)=\frac{3}{2}, \\
A_{L_{\alpha} 22} \equiv \frac{1}{2} \times 1=\frac{1}{2} .
\end{gathered}
$$

Out of four anomalous symmetries, one can form three linear combinations which are anomaly free. It is convenient to choose the following three anomaly-free symmetries $U(1)_{B / 3-L_{\alpha}}$ we mentioned earlier. Then, the anomalous symmetry $U(1)_{B+L}$ with anomaly coefficient

$$
A_{(B+L) 22}=\frac{1}{2} \times 3\left(3 \times \frac{1}{3}+1\right)=3 .
$$

From Eq. (5), one obtains the EW sphaleron effective operator

$$
\mathcal{O}_{\mathrm{SU}(2)} \sim \prod_{\alpha=1}^{3} Q_{\alpha} Q_{\alpha} Q_{\alpha} \ell_{\alpha} .
$$

The operator above violates only $U(1)_{B}$, and the interaction due to this operator is in thermal equilibrium [1] from $T_{B} \sim 2 \times 10^{12} \mathrm{GeV}$ [9] up to $T_{B-} \sim 132 \mathrm{GeV}$ [14].

The SM quark Yukawa terms are given by

$$
-\mathcal{L} \supset\left(y_{U}\right)_{a b} \overline{U_{a}} Q_{b} \epsilon H+\left(y_{D}\right)_{a b} \overline{D_{a}} Q_{b} H^{*}+\text { H.c. },
$$

where the $S U(2)_{L}$ contraction between the left-handed quark $Q_{b}$ and the Higgs $H$ doublets is shown explicitly with the $S U(2)$ antisymmetric tensor $\epsilon_{01}=-\epsilon_{10}=1$. If these terms are absent, one has a chiral symmetry $U(1)_{\chi}$ where $q_{Q_{a}}^{\chi}=-q_{U_{a}}^{\chi}=-q_{D_{a}}^{\chi} \equiv q$. Nevertheless, this chiral symmetry is anomalous with

$$
A_{\chi 33}=\frac{1}{2} \times 3(2 \times q+q+q)=6 q .
$$

From Eq. (5), one can construct the QCD sphaleron effective operator as [15]

$$
\mathcal{O}_{\mathrm{SU}(3)} \sim \prod_{\alpha=1}^{3} Q_{\alpha} Q_{\alpha} U_{\alpha}^{c} D_{\alpha}^{c}
$$

The operator above violates the chiral symmetry $U(1)_{u}$, and the interaction due to this operator is in thermal equilibrium for $T \lesssim T_{u} \sim 2 \times 10^{13} \mathrm{GeV}$ [9].

The rest of the effective symmetries in Eq. (3) are broken when the corresponding Yukawa interactions get into thermal equilibrium, starting from the one involving top Yukawa, tau Yukawa, and so on. We can estimate the temperature $T_{x}$ in which $U(1)_{x}$ is broken from the condition when the $U(1)_{x}$-violating rate is equal to the Hubble rate $\Gamma_{x}\left(T_{x}\right)=\mathcal{H}\left(T_{x}\right)$ and obtain [3]

$$
\begin{aligned}
T_{t} & \sim 1 \times 10^{15} \mathrm{GeV}, \\
T_{u} & \sim 2 \times 10^{13} \mathrm{GeV}, \\
T_{B} & \sim 2 \times 10^{12} \mathrm{GeV}, \\
T_{\tau} & \sim 4 \times 10^{11} \mathrm{GeV}, \\
T_{u-b} & \sim 3 \times 10^{11} \mathrm{GeV}, \\
T_{u-c} & \sim 2 \times 10^{10} \mathrm{GeV}, \\
T_{\mu} & \sim 10^{9} \mathrm{GeV}, \\
T_{B_{3}-B_{2}} & \sim 9 \times 10^{8} \mathrm{GeV}, \\
T_{u-s} & \sim 3 \times 10^{8} \mathrm{GeV}, \\
T_{B_{3}+B_{2}-2 B_{1}} & \sim 10^{7} \mathrm{GeV}, \\
T_{u-d} & \sim 2 \times 10^{6} \mathrm{GeV}, \\
T_{e} & \sim 3 \times 10^{4} \mathrm{GeV},
\end{aligned}
$$

and we have assumed thermalization at $T \sim 10^{15} \mathrm{GeV}$ $[16,17]$. In principle, one will need to track the evolutions of all the effective charges, starting from some initial condition. For instance, after reheating at the end of inflation with temperature $T_{\mathrm{RH}}$, we can take the initial condition to be when all the effective charges are zero. The charge density associated to each effective charge can be written as

$$
n_{\Delta x}=\sum_{i} q_{i}^{x} n_{\Delta i}
$$

where the number density asymmetry of particle $i$ is defined as $n_{\Delta i} \equiv n_{i}-n_{\bar{i}}$, where $n_{i}\left(n_{\bar{i}}\right)$ is the number density of particle $i$ (antiparticle $\bar{i}$ ). In this case, the initial condition will be $n_{\Delta x}\left(T_{\mathrm{RH}}\right)=0$ for all the charges. One should then track the evolutions of all the $n_{\Delta x}(T)$ with the Boltzmann equations including all the SM interactions. To generate some nonzero charges, the three Sakharov conditions should be fulfilled [18]:

(i) Violation of $U(1)_{x}$,

(ii) $C$ and $C P$ violation corresponding to the process violating $U(1)_{x}$,

(iii) Out-of-equilibrium condition for the process violating $U(1)_{x}$.

If the Sakharov conditions are not met for any of the charges in Eq. (3), one will always have $n_{\Delta x}=0$. If the Sakharov conditions are met for some of the $U(1)_{x}$ (this does not happen in the SM, and hence physics beyond the $\mathrm{SM}$ is required), one will have $n_{\Delta x}\left(T_{g}\right) \neq 0$, where $T_{g}$ is the temperature when the charge $n_{\Delta x}$ is being generated. If all other $U(1)_{y \neq x}$ remain effective, we have $n_{\Delta y}=0$, while for $U(1)_{y \neq x}$ which are not effective, we will necessarily have $n_{\Delta y} \propto n_{\Delta x}$. This does not necessarily imply that $n_{\Delta y} \neq 0$ since the constant of proportionality can be zero; i.e., $U(1)_{y}$ and $U(1)_{x}$ are orthogonal to each other. At $T_{B-}<T<T_{e}$, 
since $U(1)_{B}$ is not effective, we can construct the baryon charge density from Eq. (14) as

$$
n_{\Delta B}=c \sum_{\alpha} n_{\Delta\left(B / 3-L_{\alpha}\right)},
$$

where we have assumed zero hypercharge density $n_{\Delta Y}=0$. The coefficient $c$ is not zero since $B$ and $B / 3-L_{\alpha}$ are not orthogonal to each other. With the SM degrees of freedom and assuming that the EW sphaleron interaction freezes out at $132 \mathrm{GeV}$ after the EW symmetry breaking at $160 \mathrm{GeV}$ [14], we obtain

$$
n_{\Delta B}\left(T_{B-}\right)=\frac{30}{97} \sum_{a} n_{\Delta\left(B / 3-L_{a}\right)}\left(T_{B-}\right),
$$

where we have excluded the top-quark contribution.

Next, we will review briefly how to relate the number density asymmetries of the SM particles to their corresponding chemical potentials. Since all the SM particles participate in the gauge interactions, they can thermalize at a cosmic temperature $T \lesssim 10^{15} \mathrm{GeV}[16,17]$ and follow the equilibrium phase-space distribution

$$
f_{i}=\frac{1}{e^{\frac{\varepsilon_{i}-\mu_{i}}{T}}+\xi_{i}},
$$

where $\mathcal{E}_{i}$ is the energy of particle $i, \mu_{i}$ is its chemical potential, and $\xi_{i}=1(-1)$ for $i$ a fermion (boson). For gauge bosons, their numbers are not conserved, and their chemical potentials are zero. For the rest of the SM particles, due to the scatterings with the gauge bosons, the chemical potential of an antiparticle is related to the corresponding particle by a negative sign $\mu_{\bar{i}}=-\mu_{i}$. To take into account flavor correlation of particle $i$, one can generalize $\mu_{i}$ to a matrix in its flavor space. (See Appendix A for details.) In this work, since we are interested in the lepton flavor effect, we will generalize $\mu_{\ell}$ and $\mu_{E}$ to matrices in their lepton flavor spaces (see the next section).

Integrating the phase space distribution (17) over 3 -momentum, at leading order in $\left|\mu_{i}\right| / T \ll 1$ (assuming that the number density asymmetries of the SM particles are much smaller than their total number densities in the early Universe in accordance with observation), the number density asymmetries are linearly proportional to their respective chemical potentials

$$
n_{\Delta i} \equiv \int \frac{d^{3} p}{(2 \pi)^{3}}\left(f_{i}-f_{\bar{i}}\right)=\frac{T^{2}}{6} g_{i} \zeta_{i} \mu_{i}
$$

where $g_{i}$ is the gauge degrees of freedom and $\zeta_{i}=1(2)$ for $i$ a massless fermion (boson). ${ }^{3}$ To scale out the effect of

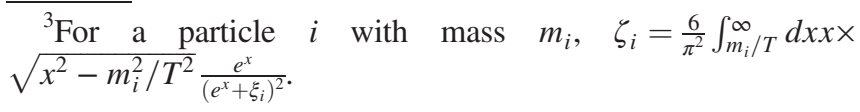

dilution purely due to the Hubble expansion, we will normalize the matrix of number densities $Y_{i} \equiv n_{i} / s$ by the cosmic entropy density $s=\frac{2 \pi^{2}}{45} g_{\star} T^{3}$ with $g_{\star}$ being the effective relativistic degrees of freedom of the Universe $\left(g_{\star}=106.75\right.$ for the SM), and we obtain

$$
Y_{\Delta i} \equiv Y_{i}-Y_{\bar{i}} \equiv Y^{\mathrm{nor}} g_{i} \zeta_{i} \frac{2 \mu_{i}}{T},
$$

where we have defined $Y^{\text {nor }} \equiv \frac{15}{8 \pi^{2} g_{\star}}$. Then, one can relate $Y_{\Delta i}$ to normalized charge density $Y_{\Delta x} \equiv n_{\Delta x} / s$ as [2]

$$
Y_{\Delta i}=\sum_{x} g_{i} \zeta_{i} \sum_{y} q_{i}^{y}\left(J^{-1}\right)_{y x} Y_{\Delta x}
$$

where

$$
J_{x y} \equiv \sum_{i} g_{i} \zeta_{i} q_{i}^{x} q_{i}^{y}
$$

The relation above is completely general (the charges are completely fixed for any given model), and the temperature dependence appears only in $\zeta_{i}$ for particles which are not massless and in $Y_{\Delta x}(T)$, which should be solved from the relevant Boltzmann equations. In the next section, we will discuss how to consider lepton flavor charges and their coherences with density matrices while treating the effects of baryons as spectators $[11,12]$.

\section{LEPTON FLAVOR EFFECT}

In the SM, we have the charged lepton Yukawa term ${ }^{4}$

$$
-\mathcal{L} \supset\left(y_{E}\right)_{\alpha \beta} \overline{E_{\alpha}} \ell_{\beta} H^{*}+\text { H.c., }
$$

where $\ell_{\beta}$ and $H$ are, respectively, the left-handed lepton and Higgs $S U(2)_{L}$ doublets, while $E_{\alpha}$ is the right-handed charged lepton $S U(2)_{L}$ singlet with family indices $\alpha$, $\beta=1,2,3$. The charged lepton Yukawa coupling can be diagonalized by two unitary matrices $U_{E}$ and $V_{E}$,

$$
\hat{y}_{E}=U_{E} y_{E} V_{E}^{\dagger},
$$

where $\hat{y}_{E}=\frac{1}{v} \operatorname{diag}\left(m_{e}, m_{\mu}, m_{\tau}\right)$ with $v \equiv\langle H\rangle=174 \mathrm{GeV}$ the Higgs vacuum expectation value and $m_{e}, m_{\mu}$, and $m_{\tau}$ are, respectively, the electron, muon, and tau lepton masses (at certain scale). In the charged lepton mass basis, which is also known as the (leptonic) flavor basis, we have $E^{\prime}=U_{E} E$ and $\ell^{\prime}=V_{E} \ell$, where they are labeled as $\ell^{\prime}=$ $\left\{\ell_{e}^{\prime}, \ell_{\mu}^{\prime}, \ell_{\tau}^{\prime}\right\}$ and $E^{\prime}=\left\{E_{e}^{\prime}, E_{\mu}^{\prime}, E_{\tau}^{\prime}\right\}$.

In this work, since we are interested in studying the flavor coherence of the lepton charges, from Eq. (19),

\footnotetext{
${ }^{4}$ In the minimal supersymmetric $\mathrm{SM}$, the corresponding term in the superpotential is $W \supset\left(y_{E}\right)_{\alpha \beta} E_{\alpha}^{c} \ell_{\beta} \epsilon H_{d}$.
} 
we will consider matrices of number density asymmetries of $\ell$ and $E$ (see Appendix A for details),

$$
Y_{\Delta \ell}=Y^{\mathrm{nor}} g_{\ell} \zeta_{\ell} \frac{2 \mu_{\ell}}{T}, \quad Y_{\Delta E}=Y^{\mathrm{nor}} g_{E} \zeta_{E} \frac{2 \mu_{E}}{T},
$$

where $Y_{\Delta \ell}, Y_{\Delta E}, \mu_{\ell}$, and $\mu_{E}$ are $3 \times 3$ Hermitian matrices in the leptonic flavor spaces (one for $\ell$ and the other for $E$ ). The diagonal elements denote the number density asymmetries in the "flavors" for any chosen basis (not necessarily the charged lepton mass basis), while the offdiagonal elements encode the correlations between the flavors. As we will see later, this generalization is necessary such that physics is independent of basis. Nevertheless, a convenient basis is usually useful to interpret the physics at hand. Including the EW sphaleron [19] and scatterings due to charged lepton Yukawa, the flavor-covariant Boltzmann equations can be written as $[5-7,9]^{5}$

$$
\begin{aligned}
s \mathcal{H} z \frac{d Y_{\Delta \ell}}{d z}= & -\frac{\gamma_{\mathrm{EW}}}{4 Y^{\text {nor }}}\left(\frac{\operatorname{Tr} Y_{\Delta \ell}}{g_{\ell} \zeta_{\ell}}+3 \frac{\operatorname{Tr} Y_{\Delta Q}}{g_{Q} \zeta_{Q}}\right) I_{3 \times 3} \\
& -\frac{\gamma_{E}}{2 Y^{\text {nor }}}\left\{y_{E}^{\dagger} y_{E}, \frac{Y_{\Delta \ell}}{g_{\ell} \zeta_{\ell}}\right\}+\frac{\gamma_{E}}{Y^{\text {nor }}} y_{E}^{\dagger} y_{E} \frac{Y_{\Delta H}}{g_{H} \zeta_{H}} \\
& +\frac{\gamma_{E}}{Y^{\text {nor }}} y_{E}^{\dagger} \frac{Y_{\Delta E}}{g_{E} \zeta_{E}} y_{E},
\end{aligned}
$$$$
s \mathcal{H} z \frac{d Y_{\Delta E}}{d z}=-\frac{\gamma_{E}}{2 Y^{\text {nor }}}\left\{y_{E} y_{E}^{\dagger}, \frac{Y_{\Delta E}}{g_{E} \zeta_{E}}\right\}-\frac{\gamma_{E}}{Y^{\text {nor }}} y_{E} y_{E}^{\dagger} \frac{Y_{\Delta H}}{g_{H} \zeta_{H}}
$$$$
+\frac{\gamma_{E}}{Y^{\text {nor }}} y_{E} \frac{Y_{\Delta \ell}}{g_{\ell} \zeta_{\ell}} y_{E}^{\dagger}
$$

where we have defined the anticommutator $\{A, B\} \equiv$ $A B+B A, z \equiv \frac{M_{\text {ref }}}{T}$ with an arbitrary reference mass scale $M_{\text {ref }}$ and $\mathcal{H}=1.66 \sqrt{g_{\star}} T^{2} / M_{\mathrm{Pl}}$ is the Hubble rate for a radiation-dominated Universe. The charged lepton Yukawa reaction density was determined in Refs. [7,20] to be $\gamma_{E} \approx 5 \times 10^{-3} \frac{T^{4}}{6}$, where thermal corrections and scatterings involving gauge fields and quark fields are taken into account. The EW sphaleron reaction density was determined in Ref. [14] to be $\gamma_{\mathrm{EW}} \approx 18 \alpha_{2}^{5} T^{4}$, where $\alpha_{2}=\frac{g_{2}^{2}}{4 \pi}$ with $g_{2}$ the weak coupling. Under arbitrary flavor rotations

$$
E \rightarrow U E, \quad \ell \rightarrow V \ell, \quad y_{E} \rightarrow U y_{E} V^{\dagger},
$$

the Boltzmann equations (25) and (26) are manifestly covariant if

\footnotetext{
${ }^{5}$ We ignore flavor oscillations which are damped by gauge interactions [6]. These equations have been derived in Refs. [6,7] using the closed time path formalism. See Appendix B for discussion on how the flavor-covariant structures can be derived from the evolution equation of a Heisenberg operator [5].
}

$$
Y_{\Delta \ell} \rightarrow V Y_{\Delta \ell} V^{\dagger}, \quad Y_{\Delta E} \rightarrow U Y_{\Delta E} U^{\dagger}
$$

The above transformations can be easily ensured when constructing the matrix of number density asymmetry as shown in Appendix A. Hence, we can use the freedom above to work in any basis while the observables; i.e., $\operatorname{Tr} Y_{\Delta \ell}$ and $\operatorname{Tr} Y_{\Delta E}$ remain unaffected by our choice of basis. For instance, we can choose $U=U_{E}$ and $V=V_{E}$, which correspond to flavor basis (23). ${ }^{6}$

In the SM, the Boltzmann equation for the evolution of total baryonic charge $Y_{\Delta B}$ is the following [19]:

$$
s \mathcal{H} z \frac{d Y_{\Delta B}}{d z}=-\frac{3 \gamma_{\mathrm{EW}}}{4 Y^{\mathrm{nor}}}\left(\frac{\operatorname{Tr} Y_{\Delta \ell}}{g_{\ell} \zeta_{\ell}}+3 \frac{\operatorname{Tr} Y_{\Delta Q}}{g_{Q} \zeta_{Q}}\right)
$$

The additional factor of 3 comes from the fact that for each scattering the change of the total baryon number is $\Delta B=3$ while for the lepton flavors we have $\Delta L_{\alpha}=1$ for each flavor. In this work, our focus is only on the lepton flavor effect, and hence we have considered $Y_{\Delta B}$ as the total baryon charge instead of matrix in the baryon flavor space. The baryon flavor effect will be considered elsewhere. Hence, we will parametrize the transitions across $T_{x}$ due to quark interactions, i.e., with $x \neq\{e, \mu, \tau\}$, as some exponential functions that we will discuss in the next section. Ignoring baryon flavor effect, let us define the charge matrix

$$
Y_{\tilde{\Delta}} \equiv \frac{1}{3} Y_{\Delta B} I_{3 \times 3}-Y_{\Delta \ell},
$$

which transforms like $Y_{\Delta \ell}$ as in Eq. (28) under flavor rotations (27). From Eqs. (25) and (29), we obtain the Boltzmann equation for $Y_{\tilde{\Delta}}$ as follows:

$$
\begin{aligned}
s \mathcal{H} z \frac{d Y_{\tilde{\Delta}}}{d z}= & \frac{\gamma_{E}}{2 Y^{\text {nor }}}\left\{y_{E}^{\dagger} y_{E}, \frac{Y_{\Delta \ell}}{g_{\ell} \zeta_{\ell}}\right\}-\frac{\gamma_{E}}{Y^{\text {nor }}} y_{E}^{\dagger} y_{E} \frac{Y_{\Delta H}}{g_{H} \zeta_{H}} \\
& -\frac{\gamma_{E}}{Y^{\text {nor }}} y_{E}^{\dagger} \frac{Y_{\Delta E}}{g_{E} \zeta_{E}} y_{E} .
\end{aligned}
$$

Now, we only need to solve (26) and (31), treating $Y_{\tilde{\Delta}}$ and $Y_{\Delta E}$ as the only independent variables.

One could have defined the $B / 3-L_{a}$ charge matrix

$$
Y_{\Delta} \equiv \frac{1}{3} Y_{\Delta B} I_{3 \times 3}-Y_{\Delta \ell}-Y_{\Delta E}
$$

where one would have to keep in mind that $Y_{\Delta \ell}$ and $Y_{\Delta E}$ transform differently as in Eq. (28). Clearly, the physics will remain the same, but in order to avoid remembering the different transformations within $Y_{\Delta}$, we will resort to using $Y_{\tilde{\Delta}}$. Nevertheless, it is instructive to look at the Boltzmann

\footnotetext{
${ }^{6}$ Including the Renormalized Group Evolution (RGE) of charged lepton Yukawa coupling, $U_{E}$ and $V_{E}$ will in general be scale dependent.
} 
equation for $Y_{\Delta}$ in the flavor basis where we can construct from Eqs. (25), (26), and (29) as follows:

$$
\begin{aligned}
s \mathcal{H} z \frac{d Y_{\Delta}}{d z}= & \frac{\gamma_{E}}{2 Y^{\text {nor }}}\left\{\hat{y}_{E}^{2}, \frac{Y_{\Delta \ell}}{g_{\ell} \zeta_{\ell}}\right\}-\frac{\gamma_{E}}{Y^{\text {nor }}} \hat{y}_{E} \frac{Y_{\Delta E}}{g_{E} \zeta_{E}} \hat{y}_{E} \\
& +\frac{\gamma_{E}}{2 Y^{\text {nor }}}\left\{\hat{y}_{E}^{2}, \frac{Y_{\Delta E}}{g_{E} \zeta_{E}}\right\}-\frac{\gamma_{E}}{Y^{\text {nor }}} \hat{y}_{E} \frac{Y_{\Delta \ell}}{g_{\ell} \zeta_{\ell}} \hat{y}_{E} \\
= & -\frac{\gamma_{E}}{2 Y^{\text {nor }}}\left[\hat{y}_{E},\left[\hat{y}_{E}, \frac{Y_{\Delta \ell}}{g_{\ell} \zeta_{\ell}}+\frac{Y_{\Delta E}}{g_{E} \zeta_{E}}\right]\right] .
\end{aligned}
$$

In the last step above, we have defined the commutator $[A, B] \equiv A B-B A$. One can easily check that in the flavor basis the double anticommutator term projects out only the off-diagonal entries of $Y_{\Delta \ell}$ and $Y_{\Delta E}$. Hence, $\operatorname{Tr} Y_{\Delta}$ remains a constant as it should be since the SM interactions do not break $B-L$. Clearly, the same conclusion holds also in any other basis. While it is not necessary to work in flavor basis, it makes the interpretation easier since in the flavor basis one can identify the diagonal elements of $Y_{\Delta}$ as the flavor charges $Y_{\Delta\left(B / 3-L_{e}\right)}, Y_{\Delta \Delta\left(B / 3-L_{\mu}\right)}$, and $Y_{\Delta\left(B / 3-L_{\tau}\right)}$. In Eq. (33), it is apparent that for a consistent description of evolution of lepton flavor charges which is basis independent both $Y_{\Delta \ell}$ and $Y_{\Delta E}$ need to be described by density matrices: if off-diagonal terms of $Y_{\Delta \ell}$ are induced, offdiagonal terms for $Y_{\Delta E}$ will be induced as well and vice versa.

In the rest of the work, we will use Eqs. (26) and (31), which are valid in any basis. Including new physics interactions that generate either $Y_{\Delta E}$ and/or $Y_{\tilde{\Delta}}$ in the two Boltzmann equations, from Eq. (16), the final baryon asymmetry will be frozen at $T_{B-}$ to be

$$
Y_{\Delta B}\left(T_{B-}\right)=\left.\frac{30}{97}\left(\operatorname{Tr} Y_{\tilde{\Delta}}-\operatorname{Tr} Y_{\Delta E}\right)\right|_{T=T_{B-}} .
$$

Next, we will write down the relations between $Y_{\Delta \ell}$ and $Y_{\Delta H}$ in terms of $Y_{\tilde{\Delta}}$ and $Y_{\Delta E}$ for the SM and the SM augmented with arbitrary scalar fields carrying nonzero hypercharges.

\section{A. Standard Model}

With the SM field content, from Eq. (20), we obtain ${ }^{7}$

$$
\begin{gathered}
\left(Y_{\Delta \ell}\right)_{\alpha \alpha}=\frac{2}{15} c_{B} \operatorname{Tr} Y_{\tilde{\Delta}}-\left(Y_{\tilde{\Delta}}\right)_{\alpha \alpha}, \\
Y_{\Delta H}=-c_{H}\left(\operatorname{Tr} Y_{\tilde{\Delta}}-2 \operatorname{Tr} Y_{\Delta E}\right),
\end{gathered}
$$

where $c_{B}$ and $c_{H}$ are coefficients which vary with temperature. In obtaining the expressions above, we have assumed all effective charges in Eq. (3), except $\left(Y_{\tilde{\Delta}}\right)_{\alpha \alpha}$ and $\left(Y_{\Delta E}\right)_{\alpha \alpha}$, to be zero. Comparing with Eq. (30), one recognizes

\footnotetext{
${ }^{7}$ The number asymmetries of quark fields in term of $Y_{\tilde{\Delta}}$ and $Y_{\Delta E}$ are collected in Appendix C.
}

$\frac{B}{3}=\frac{2}{15} c_{B} \operatorname{Tr} Y_{\tilde{\Delta}}$. The relations above are completely general and capture all the spectator effects in the SM. At $T>T_{B} \sim 2 \times 10^{12} \mathrm{GeV}$ when the EW sphaleron interaction is out of equilibrium, we have $c_{B}=0$, while at $T<T_{B}$ when the baryon number is no longer conserved, we have $c_{B}=1$. This shows that an asymmetry in the lepton sector is being shared with the baryon sector and vice versa at $T<T_{B}$. To capture this effect in a continuous manner, one should consider $Y_{\Delta \ell_{a}}=\frac{B}{3}-Y_{\tilde{\Delta}_{a}}$ and include the Boltzmann equation for $Y_{\Delta B}$ in Eq. (29) and then solve for $c_{B}(T)$. To within percent-level precision, one can use the fitting function ${ }^{8}$

$$
c_{B}(T)=1-e^{-\frac{T_{B}}{T}},
$$

where $T_{B}=2.3 \times 10^{3} \mathrm{GeV}$. In Appendix D, we discuss how to determine a precise value of $T_{B}$.

The rest of the spectator effects pertaining to quark sector are encapsulated in the coefficient $c_{H}$ with

$$
c_{H}(T)=\left\{\begin{array}{ll}
1 & T>T_{t} \\
\frac{2}{3} & T_{u}<T<T_{t} \\
\frac{14}{23} & T_{u-b}<T<T_{u} \\
\frac{2}{5} & T_{u-c}<T<T_{u-b} \\
\frac{4}{13} & T_{B_{3}-B_{2}}<T<T_{u-c} \\
\frac{3}{10} & T_{u-s}<T<T_{B_{3}-B_{2}} \\
\frac{1}{4} & T_{u-d}<T<T_{u-s} \\
\frac{2}{11} & T<T_{u-d}
\end{array} .\right.
$$

In the equation above, we can see explicitly that the asymmetry carried by the Higgs is diluted as more charges come into equilibrium. Since the transitions due to the rate $\Gamma \propto T$ as compared to the Hubble rate $H \propto T^{2}$ always have an exponential behavior, one can parametrize the transitions with the following function:

$$
\begin{aligned}
c_{H}(T)= & \left(\frac{2}{3}+\frac{1}{3} e^{-\frac{T_{t}}{T}}\right)-\left(\frac{2}{3}-\frac{14}{23}\right)\left(1-e^{-\frac{T_{u}}{T}}\right) \\
& -\left(\frac{14}{23}-\frac{2}{5}\right)\left(1-e^{-\frac{T_{u-b}}{T}}\right)-\left(\frac{2}{5}-\frac{4}{13}\right)\left(1-e^{-\frac{T_{u-c}}{T}}\right) \\
& -\left(\frac{4}{13}-\frac{3}{10}\right)\left(1-e^{-\frac{T_{B_{3}-B_{2}}}{T}}\right) \\
& -\left(\frac{3}{10}-\frac{1}{4}\right)\left(1-e^{-\frac{T_{u-s}}{T}}\right)-\left(\frac{1}{4}-\frac{2}{11}\right)\left(1-e^{-\frac{T_{u-d}}{T}}\right) .
\end{aligned}
$$

\footnotetext{
${ }^{8}$ One can also use a theta function $c_{B}(T)=\theta\left(T-T_{B}\right)$, keeping in mind that the effect can be of the order of 1 if leptogenesis happens around $T_{B}$.
} 
For the purpose of this work, we use the transition temperatures as shown in Eq. (13). Precise determination of the transition temperatures can be carried out following the procedure shown in Appendix D.

From the definition of $Y_{\tilde{\Delta}}$ in Eq. (30), the off-diagonal terms $\alpha \neq \beta$ are

$$
\left(Y_{\tilde{\Delta}}\right)_{\alpha \beta}=-\left(Y_{\Delta \ell}\right)_{\alpha \beta} .
$$

Hence, we can rewrite the matrix $Y_{\Delta \ell}$ as

$$
Y_{\Delta \ell}=\frac{2}{15} c_{B} I_{3 \times 3} \operatorname{Tr} Y_{\tilde{\Delta}}-Y_{\tilde{\Delta}} .
$$

\section{B. Standard Model with additional scalar fields}

If one introduces additional scalar fields $\phi_{i}$ with hypercharge $q_{\phi_{i}}^{Y}$ to the system, Eq. (35) remains the same, while Eq. (36) changes to

$$
Y_{\Delta H}=-c_{H}\left(\operatorname{Tr} Y_{\tilde{\Delta}}-2 \operatorname{Tr} Y_{\Delta E}+2 \sum_{i} q_{\phi_{i}}^{Y} Y_{\Delta \phi_{i}}\right)
$$

where $Y_{\Delta \phi_{i}}$ defined in Eq. (19) takes into account additional gauge multiplicity $g_{\phi_{i}}$ as well as mass of $\phi_{i}$ in $\zeta_{\phi_{i}}$ (implicitly, we have assumed $\phi_{i}$ to be in kinetic equilibrium but not necessarily in chemical equilibrium). The relation above is general, independently of whether $\phi_{i}$ are in chemical equilibrium or not. If some of the $\phi_{i}$ do not achieve chemical equilibrium, one will have effective $U(1)_{\phi_{i}}$ in which $Y_{\Delta \phi_{i}}$ remains constant. Otherwise, the evolution of $Y_{\Delta \phi_{i}}$ will have to be described by the corresponding Boltzmann equation.

For instance, for type-II seesaw leptogenesis with a heavy triplet Higgs $\mathcal{T}$ with hypercharge $q_{\mathcal{T}}^{Y}=1$, one can apply Eq. (42) and obtain

$$
Y_{\Delta H}=-c_{H}\left(\operatorname{Tr} Y_{\tilde{\Delta}}-2 \operatorname{Tr} Y_{\Delta E}+2 Y_{\Delta \mathcal{T}}\right) .
$$

\section{APPLICATIONS}

Now, we will apply the flavor-covariant Boltzmann equations (26) and (31) to some well-motivated leptogenesis scenarios. One just needs the general expressions (41) and (42) to close the equations. Even for leptogenesis models involving quarks, one can use the general relations in Appendix C (ignoring baryon flavor effect). Hence, one no longer needs to solve for flavor matrices for a particular model and which hold only in a particular temperature regime as has been done, for example, in Refs. [21,22]. In the first example, we will apply the formalism to type-I leptogenesis, while in the second example, we will apply it to type-II leptogenesis where flavor-covariant formalism is indispensable as first pointed out in Ref. [22]. In particular, we will demonstrate that the results obtained are independent of basis, showing that it is necessary to take into account flavor correlation in both $\ell$ and $E$. In other words, it is inconsistent to consider flavor correlation only in $\ell$ or only in $E$.

\section{A. Type-I leptogenesis}

In the type-I seesaw model, the SM is extended by righthanded neutrinos $N_{i}$ as

$$
-\mathcal{L} \supset \frac{1}{2} M_{i} \overline{N_{i}} N_{i}^{c}+y_{i \alpha} \overline{N_{i}} \ell_{\alpha} \epsilon H+\text { H.c. },
$$

where $M_{i}$ is the Majorana mass of $N_{i}$ and we will work in the arbitrary basis where $y_{E}$ is not necessarily diagonal. While two generations of $N_{i}$ are already sufficient to explain neutrino oscillation data, as an example, we will consider three generations $i=1,2,3$.

After the EW symmetry breaking with $v \equiv\langle H\rangle=$ $174 \mathrm{GeV}$, the light neutrino mass matrix for $|y| v \ll M_{i}$ is

$$
m_{\nu}^{\mathrm{I}}=-v^{2} y^{T} M^{-1} y
$$

where $M=\operatorname{diag}\left(M_{1}, M_{2}, M_{3}\right)$. The mass matrix can be diagonalized with $U_{\nu}^{T} m_{\nu} U_{\nu}=\hat{m} \equiv \operatorname{diag}\left(m_{1}, m_{2}, m_{3}\right)$, where $U_{\mathrm{PMNS}}=V_{E} U_{\nu}$ is identified with the leptonic mixing matrix.

For type-I leptogenesis, an asymmetry is generated through the $C P$-violating decays $N_{i} \rightarrow \ell_{\alpha} H$. In addition to the Boltzmann equation for $Y_{N_{i}}$,

$$
s \mathcal{H} z \frac{d Y_{N_{i}}}{d z}=-\gamma_{N_{i}}\left(\frac{Y_{N_{i}}}{Y_{N_{i}}^{\mathrm{eq}}}-1\right),
$$

where we have defined $z \equiv M_{1} / T$, we have to append to the right-hand side of Eq. (31) a source and washout terms, respectively, given by [10]

$$
\begin{gathered}
S^{\mathrm{I}} \equiv-\sum_{i} \epsilon_{i} \gamma_{N_{i}}\left(\frac{Y_{N_{i}}}{Y_{N_{i}}^{\mathrm{eq}}}-1\right), \\
W^{\mathrm{I}} \equiv \frac{1}{2} \sum_{i} \frac{\gamma_{N_{i}}}{Y^{\text {nor }}}\left(\frac{1}{2}\left\{P_{i}, \frac{Y_{\Delta \ell}}{g_{\ell} \zeta_{\ell}}\right\}+P_{i} \frac{Y_{\Delta H}}{g_{H} \zeta_{H}}\right),
\end{gathered}
$$

where to close the equations we apply Eqs. (36) and (41). Assuming the Maxwell-Boltzmann distribution for $N_{i}$, we have $Y_{N_{i}}^{\text {eq }}=\frac{45}{2 \pi^{4} g_{\star}} \frac{M_{i}^{2}}{T^{2}} \mathcal{K}_{2}\left(\frac{M_{i}}{T}\right)$, with $\mathcal{K}_{n}(x)$ the modified Bessel function of the second kind of order $n$, and the decay reaction density $\gamma_{N_{i}}$ is given by 


$$
\gamma_{N_{i}}=s Y_{N_{i}}^{\mathrm{eq}} \Gamma_{N_{i}} \frac{\mathcal{K}_{1}\left(M_{i} / T\right)}{\mathcal{K}_{2}\left(M_{i} / T\right)}
$$

with $\Gamma_{N_{i}}=\frac{\left(y y^{\dagger}\right)_{i i} M_{i}}{8 \pi}$ the total decay width of $N_{i}{ }^{9}$

The matrix of $C P$-violation parameter $\epsilon_{i}$ and flavor rotation matrix $P_{i}$ are, respectively, given by [10]

$$
\left(\epsilon_{i}\right)_{\alpha \beta}=\frac{1}{16 \pi} \frac{i}{\left(y y^{\dagger}\right)_{i i}} \sum_{j \neq i}\left[\left(y y^{\dagger}\right)_{j i} y_{j \beta} y_{i \alpha}^{*}-\left(y y^{\dagger}\right)_{i j} y_{i \beta} y_{j \alpha}^{*}\right] g\left(\frac{M_{j}^{2}}{M_{i}^{2}}\right)+\frac{1}{16 \pi} \frac{i}{\left(y y^{\dagger}\right)_{i i}} \sum_{j \neq i}\left[\left(y y^{\dagger}\right)_{i j} y_{j \beta} y_{i \alpha}^{*}-\left(y y^{\dagger}\right)_{j i} y_{i \beta} y_{j \alpha}^{*}\right] \frac{M_{i}^{2}}{M_{i}^{2}-M_{j}^{2}},
$$

$$
P_{i}=\frac{1}{\left(y y^{\dagger}\right)_{i i}}\left(\begin{array}{ccc}
\left|y_{i e}\right|^{2} & y_{i e}^{*} y_{i \mu} & y_{i e}^{*} y_{i \tau} \\
y_{i e} y_{i \mu}^{*} & \left|y_{i \mu}\right|^{2} & y_{i \mu}^{*} y_{i \tau} \\
y_{i e} y_{i \tau}^{*} & y_{i \mu} y_{i \tau}^{*} & \left|y_{i \tau}\right|^{2}
\end{array}\right) .
$$

Under flavor rotations (27) and (28), we have

$$
\epsilon_{i} \rightarrow V \epsilon_{i} V^{\dagger}, \quad P_{i} \rightarrow V P_{i} V^{\dagger}
$$

and the whole Boltzmann equation for $Y_{\tilde{\Delta}}$ remains flavor covariant as required.

For illustration, we choose the best-fit point from Ref. [24] for the $\mathrm{SO}(10)$ model with Higgs content $10_{H}+\overline{126}_{H}$ for the Yukawa sector with no-RGE

$$
\begin{gathered}
y=\left(\begin{array}{ccc}
(2.508-1.101 i) \times 10^{-4} & (1.224-5.313 i) \times 10^{-4} & (-1.988+0.646 i) \times 10^{-2} \\
(1.893+0.0359 i) \times 10^{-3} & (-2.100+20.365 i) \times 10^{-3} & (-8.560+1.384 i) \times 10^{-2} \\
(1.446-9.365 i) \times 10^{-3} & (2.217+1.373 i) \times 10^{-2} & 0.1356+0.4602 i
\end{array}\right), \\
y_{E}=\left(\begin{array}{ccc}
(1.0077+1.0449 i) \times 10^{-5} & (-3.8245+0.0226 i) \times 10^{-5} & (-3.2332-1.8088 i) \times 10^{-4} \\
(-3.8245+0.0226 i) \times 10^{-5} & (5.2064-2.2026 i) \times 10^{-4} & (8.0184-7.4693 i) \times 10^{-4} \\
(-3.2332-1.8088 i) \times 10^{-4} & (8.0184-7.4693 i) \times 10^{-4} & (8.5102+4.4337 i) \times 10^{-3}
\end{array}\right) \\
M=\left\{1.445 \times 10^{10}, 7.244 \times 10^{11}, 5.663 \times 10^{12}\right\} \mathrm{GeV} .
\end{gathered}
$$

We will solve the Boltzmann equations in the original basis (as above) and in the flavor basis where $y_{E}$ is diagonalized through a flavor rotation as in Eq. (23) assuming zero initial abundance $Y_{N_{i}}\left(z_{i}\right)=0$ with $z_{i}=10^{-4}$.

In Fig. 1, we show the numerical solutions comparing the results in the nonflavor basis $y_{E}$ (solid curves) and in the flavor basis $\hat{y}_{E}$ (dashed curves). In the top row, we show the diagonal elements of $\left|Y_{\tilde{\Delta}}\right|$ and $\left|Y_{\Delta E}\right|$, while in the bottom

\footnotetext{
${ }^{9}$ Here, we consider only decay and inverse decay. We have ignored the helicities of $N_{i}$ and scattering processes which will be relevant for leptogenesis in the weak washout regime $\Gamma_{N_{i}} / \mathcal{H}\left(T=M_{i}\right) \ll 1$ since in this case the physics at $T \gg M_{i}$ will play a relevant role [23]. We have also assumed $N_{i}$ to be wellseparated states $\left|M_{i}-M_{j}\right| \gg \Gamma_{N_{i, j}}$ such that the effect of $N_{i}$ oscillations is not relevant. Otherwise, one should use the flavorcovariant formalism which also includes the flavor of $N_{i}$ [8].
}

row, we show their off-diagonal elements (they are Hermitian matrices). Here, we see that independent of basis, once off-diagonal elements of $Y_{\tilde{\Delta}}$ develop from leptogenesis, unavoidably, off-diagonal elements of $Y_{\Delta E}$ will be induced as well. In the flavor basis $\hat{y}_{E}$, the offdiagonal elements start to become suppressed at various temperatures as the charged lepton Yukawa interactions subsequently get into thermal equilibrium and finally at $z \gtrsim 100,\left(Y_{\tilde{\Delta}}\right)_{12}$ and $\left(Y_{\Delta E}\right)_{12}$ start to become suppressed, indicating a transition to the three-flavor regime.

In Fig. 2, top row, we plot $\left|\operatorname{Tr} Y_{\tilde{\Delta}}\right|$ and $\left|\operatorname{Tr} Y_{\Delta E}\right|$ in the two different bases: nonflavor basis $y_{E}$ (red solid curve) and flavor basis $\hat{y}_{E}$ (blue dashed curve). Reassuringly, $\operatorname{Tr} Y_{\tilde{\Delta}}$ and $\operatorname{Tr} Y_{\Delta E}$ are basis independent, although the entries of $Y_{\tilde{\Delta}}$ and $Y_{\Delta E}$ differ among the two bases by the flavor rotations as in Eq. (28) with $V=V_{E}$ and $U=V_{E}^{*}$ since $y_{E}$ is symmetric. Clearly, the physics is invariant under basis 

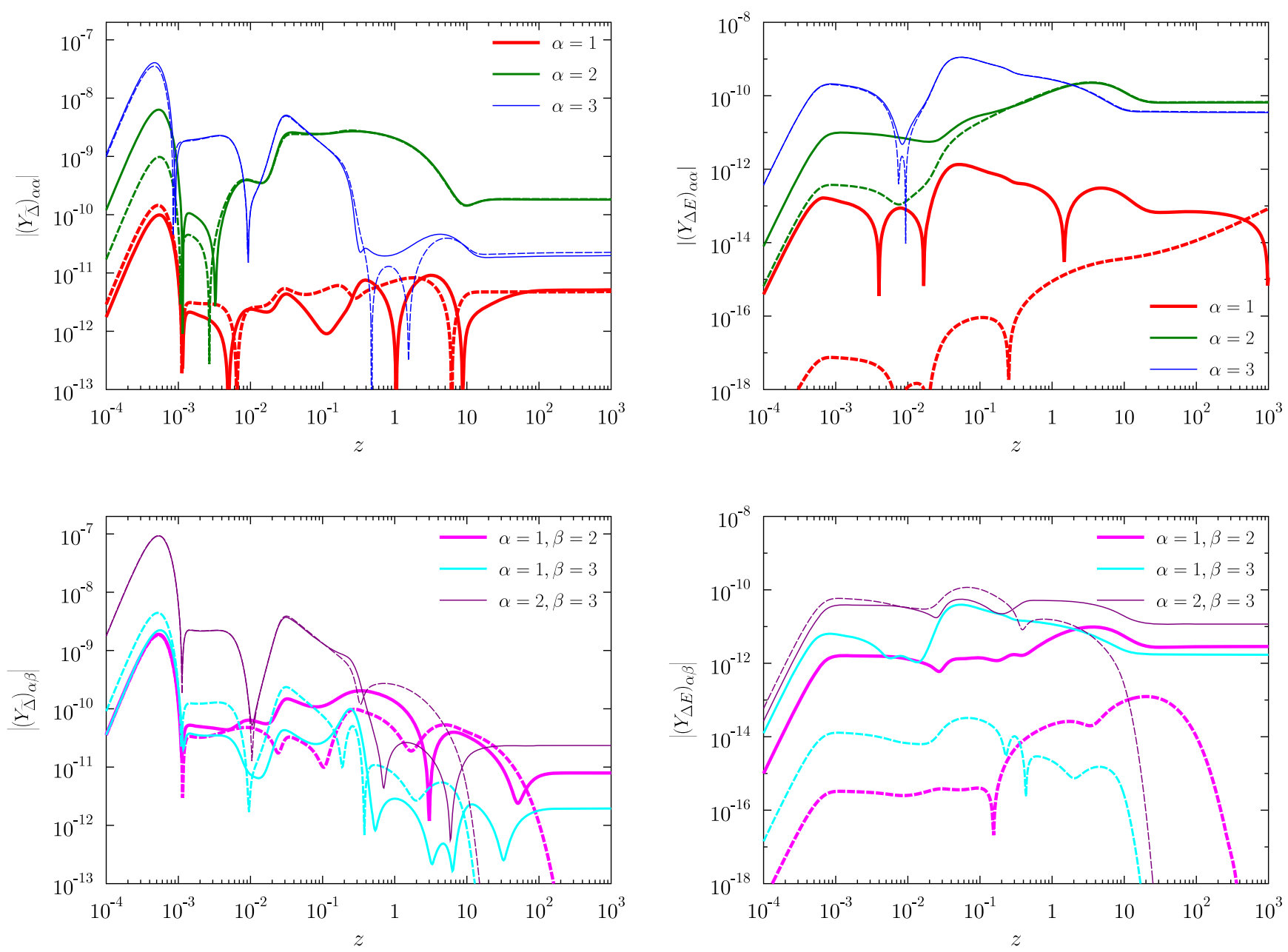

FIG. 1. Numerical solutions for type-I leptogenesis. In the top row, we plot the diagonal elements of $\left|Y_{\tilde{\Delta}}\right|$ and $\left|Y_{\Delta E}\right|$ in the two different bases: nonflavor basis $y_{E}$ (solid curves) and flavor basis $\hat{y}_{E}$ (dashed curves). In the bottom row, we plot the off-diagonal elements of $\left|Y_{\tilde{\Delta}}\right|$ and $\left|Y_{\Delta E}\right|$ in the $y_{E}$ (solid curves) and $\hat{y}_{E}$ (dashed curves) bases. Colors (thickness) denote different matrix elements as indicated in the plots. See the text for further discussions.

transformation, and the benefit of the flavor basis is to help us to interpret the results. For instance, we can read the diagonal entries of $Y_{\tilde{\Delta}}$ and $Y_{\Delta E}$ in the $\hat{y}_{E}$ basis as the flavor charges in the $e, \mu, \tau$ (red, blue and green dashed curves in the top row of the Fig. 1) and also deduce when the system transits to a different flavor regime from the suppression of off-diagonal entries. In the bottom plot of Fig. 2, we see that, while $\left|Y_{\Delta(B-L)}\right|=\left|\operatorname{Tr} Y_{\tilde{\Delta}}-\operatorname{Tr} Y_{\Delta E}\right|$ is conserved at the end of leptogenesis $z \gtrsim 10,\left|\operatorname{Tr} Y_{\tilde{\Delta}}\right|$ and $\left|\operatorname{Tr} Y_{\Delta E}\right|$, not being conserved charges, continue to evolve. For a final remark, the final baryon asymmetry produced in this example is $Y_{\Delta B}\left(T_{B-}\right)=6.1 \times 10^{-11}$, consistent in sign but smaller than the observed value by about $30 \% .^{10}$

\footnotetext{
${ }^{10}$ This can be compared with Ref. [25], which also obtained a final baryon asymmetry, which is of the right sign but a factor of a few smaller than the observed baryon asymmetry. Besides the improved treatment discussed in the work, we also correct the wrong basis used in Ref. [25].
}

\section{B. Type-II leptogenesis}

In the type-II seesaw model, the SM is extended by a massive triplet scalar $\mathcal{T}$ under $S U(2)_{L}$ with hypercharge $q_{\mathcal{T}}^{Y}=1$ as

$-\mathcal{L} \supset M_{\mathcal{T}}^{2} \operatorname{Tr}\left(\mathcal{T}^{\dagger} \mathcal{T}\right)+\frac{1}{2}\left(f_{\alpha \beta} \overline{\ell_{\alpha}^{c}} \epsilon \mathcal{T} \ell_{\beta}+\mu H^{T} \epsilon \mathcal{T}^{\dagger} H+\right.$ H.c. $)$,

where

$$
\mathcal{T}=\left(\begin{array}{cc}
\frac{1}{\sqrt{2}} \mathcal{T}^{+} & \mathcal{T}^{++} \\
\mathcal{T}^{0} & -\frac{1}{\sqrt{2}} \mathcal{T}^{+}
\end{array}\right)
$$

Since $\mathcal{T}$ couples to two lepton doublets which in general do not align in flavor space, one needs to describe them with density matrix as first pointed out in Ref. [22]. 

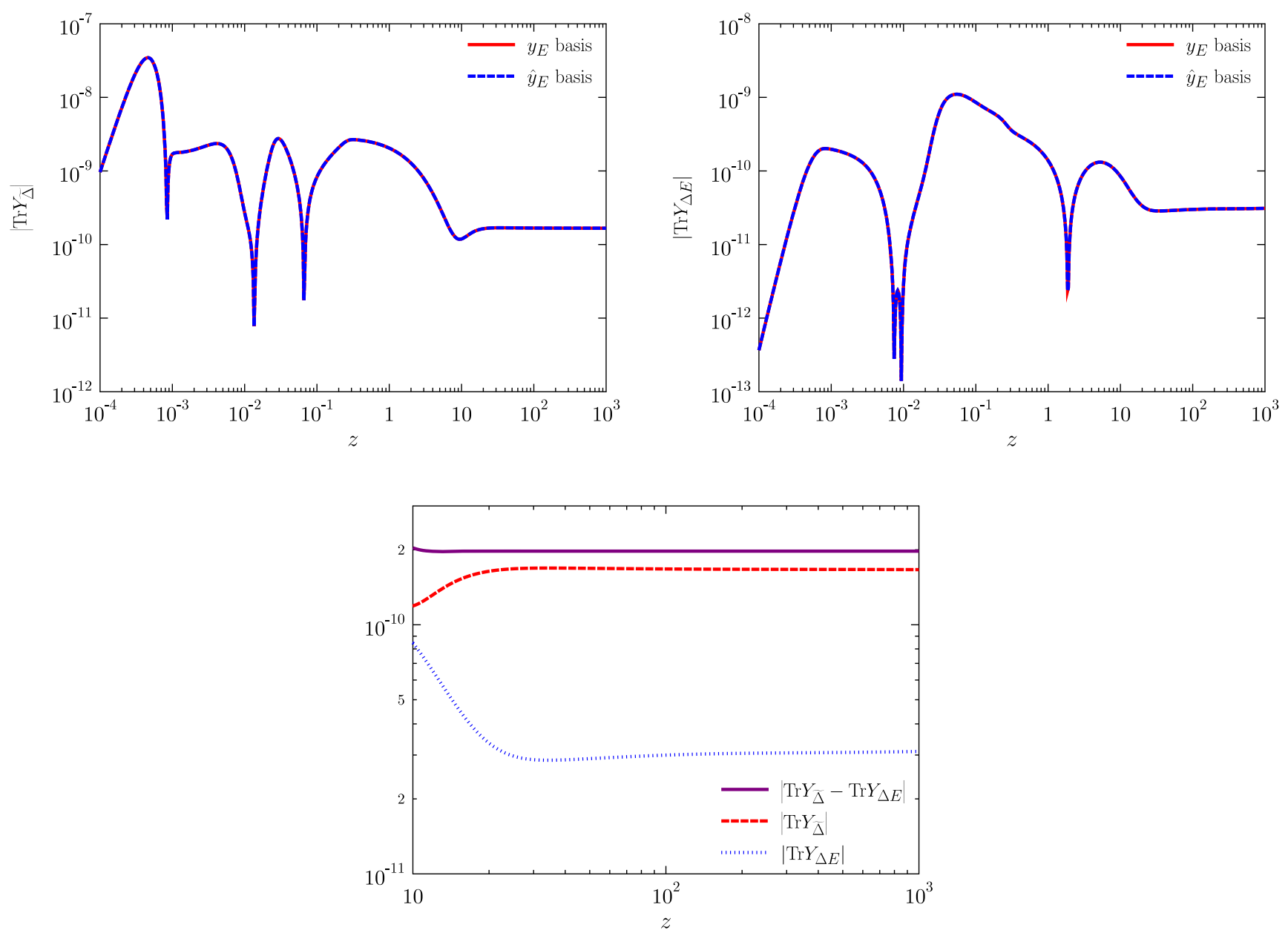

FIG. 2. Numerical solutions for type-I leptogenesis. In the top row, we plot $\left|\operatorname{Tr} Y_{\tilde{\Delta}}\right|$ and $\left|\operatorname{Tr} Y_{\Delta E}\right|$ in the two different bases: nonflavor basis $y_{E}$ (red solid curve) and flavor basis $\hat{y}_{E}$ (blue dashed curve). In the bottom plot, we plot $\left|\operatorname{Tr} Y_{\tilde{\Delta}}-\operatorname{Tr} Y_{\Delta E}\right|$ (purple solid curve), $\left|\operatorname{Tr} Y_{\tilde{\Delta}}\right|$ (red dashed curve), and $\left|\operatorname{Tr} Y_{\Delta E}\right|$ (blue dotted curve) for $z>10$. See the text for further discussions.

The $C P$ violation in the decays of $\mathcal{T}^{\dagger} \rightarrow \ell_{\alpha} \ell_{\beta}$ and $\mathcal{T} \rightarrow H H$ can arise at one-loop level from the contribution of heavier particles of mass scale $\Lambda \gg M_{T}$, which generate the Weinberg operator below $\Lambda$,

$$
\mathcal{L}_{\text {eff }}=\frac{1}{4} \frac{\kappa_{\alpha \beta}}{\Lambda} \bar{\ell}_{\alpha}^{c} \epsilon H H^{T} \epsilon \ell_{\beta}+\text { H.c. }
$$

After the EW symmetry breaking, the light neutrino mass receives contributions from integrating out the scalar triplet $T$ as well as the Weinberg operator as

$$
m_{\nu}^{\mathrm{II}}=m_{\mathcal{T}}+m_{\mathcal{H}},
$$

where

$$
\begin{gathered}
m_{\mathcal{T}} \equiv \frac{1}{2} \mu f \frac{v^{2}}{M_{T}^{2}}, \\
m_{\mathcal{H}} \equiv \frac{1}{2} \kappa \frac{v^{2}}{\Lambda} .
\end{gathered}
$$

In the following, we will utilize the interaction terms derived in Ref. [22] but include only decay, inverse decay, and gauge scattering processes (other scattering effects are negligible in the parameter space we will consider below). The Boltzmann equations to describe the evolution of $Y_{\Sigma \mathcal{T}} \equiv Y_{\mathcal{T}}+Y_{\mathcal{T}^{\dagger}}$ and $Y_{\Delta \mathcal{T}} \equiv Y_{\mathcal{T}}-Y_{\mathcal{T}^{\dagger}}$ are [22]

$s \mathcal{H} z \frac{d Y_{\Sigma \mathcal{T}}}{d z}=-\gamma_{D}\left(\frac{Y_{\Sigma \mathcal{T}}}{Y_{\Sigma \mathcal{T}}^{\mathrm{eq}}}-1\right)-2 \gamma_{A}\left(\frac{Y_{\Sigma \mathcal{T}}^{2}}{Y_{\Sigma \mathcal{T}}^{\mathrm{eq}, 2}}-1\right)$,

$s \mathcal{H} z \frac{d Y_{\Delta \mathcal{T}}}{d z}=-\gamma_{D}\left(\frac{Y_{\Delta \mathcal{T}}}{Y_{\Sigma \mathcal{T}}^{\mathrm{eq}}}+B_{\ell} \frac{\operatorname{Tr}\left(f f^{\dagger} Y_{\Delta \ell}\right)}{\operatorname{Tr}\left(f f^{\dagger}\right) Y^{\mathrm{nor}} g_{\ell} \zeta_{\ell}}\right.$

$$
\left.-B_{H} \frac{Y_{\Delta H}}{Y^{\text {nor }} g_{H} \zeta_{H}}\right)
$$

where we have defined $z \equiv M_{\mathcal{T}} / T$ and to close the equations we apply Eqs. (41) and (43). The branching ratios for the decays of $\mathcal{T}$ to lepton doublets and Higgses are, respectively, 


$$
\begin{aligned}
& B_{\ell}=\frac{\operatorname{Tr}\left(f f^{\dagger}\right)}{\operatorname{Tr}\left(f f^{\dagger}\right)+\frac{|\mu|^{2}}{M_{T}^{2}}}, \\
& B_{H}=\frac{\frac{|\mu|^{2}}{M_{T}^{2}}}{\operatorname{Tr}\left(f f^{\dagger}\right)+\frac{|\mu|^{2}}{M_{T}^{2}}} .
\end{aligned}
$$

For the generation of $Y_{\tilde{\Delta}}$, we have to append to the righthand side of Eq. (31) a source and washout terms, respectively, given by [22]

$$
\begin{gathered}
S^{\mathrm{II}} \equiv-\epsilon \gamma_{D}\left(\frac{Y_{\Sigma \mathcal{T}}}{Y_{\mathcal{T}}^{\mathrm{eq}}}-1\right) \\
W^{\mathrm{II}} \equiv \frac{2 \gamma_{D}}{\operatorname{Tr}\left(f f^{\dagger}\right)+\frac{|\mu|^{2}}{M_{\mathcal{T}}^{2}}}\left[\left(f f^{\dagger}\right) \frac{Y_{\Delta \mathcal{T}}}{Y_{\mathcal{T}}^{\mathrm{eq}}}\right. \\
\left.+\frac{1}{4 Y^{\mathrm{nor}} g_{\ell} \zeta_{\ell}}\left(2 f Y_{\Delta \ell}^{T} f^{\dagger}+f f^{\dagger} Y_{\Delta \ell}+Y_{\Delta \ell} f f^{\dagger}\right)\right],
\end{gathered}
$$

where the matrix of the $C P$-violation parameter is

$$
\epsilon=\frac{i}{8 \pi} \frac{M_{T}}{v^{2}} \sqrt{B_{\ell} B_{H}} \frac{m_{\mathcal{T}} m_{\mathcal{H}}^{\dagger}-m_{\mathcal{H}} m_{\mathcal{T}}^{\dagger}}{\sqrt{\operatorname{Tr}\left(m_{\mathcal{T}}^{\dagger} m_{\mathcal{T}}\right)}} .
$$

Assuming Maxwell-Boltzmann distribution for $\mathcal{T}$, we have $Y_{\Sigma \mathcal{T}}^{\mathrm{eq}}=Y_{\mathcal{T}}^{\mathrm{eq}}+Y_{\mathcal{T}^{\dagger}}^{\mathrm{eq}}=\frac{135}{2 \pi^{4} g_{\star}} z^{2} \mathcal{K}_{2}(z)$, and the decay reaction density $\gamma_{D}$ is given by

$$
\gamma_{D}=s Y_{\Sigma \mathcal{T}}^{\mathrm{eq}} \Gamma_{\mathcal{T}} \frac{\mathcal{K}_{1}(z)}{\mathcal{K}_{2}(z)}
$$

where the total decay width is

$$
\Gamma_{\mathcal{T}}=\frac{M_{\mathcal{T}}}{32 \pi}\left[\operatorname{Tr}\left(f f^{\dagger}\right)+\frac{|\mu|^{2}}{M_{\mathcal{T}}^{2}}\right]
$$

Finally, assuming Maxwell-Boltzmann distributions for all the particles, the gauge scattering reaction density for $\mathcal{T} \mathcal{T}^{\dagger} \leftrightarrow \psi \bar{\psi}$, where $\psi$ refers to the SM fields, is

$$
\gamma_{A}=\frac{M_{\mathcal{T}}^{4}}{64 \pi^{4} z} \int_{4}^{\infty} d x \sqrt{x} \mathcal{K}_{1}(z \sqrt{x}) \hat{\sigma}_{A}(x)
$$

where the reduced cross section is given by [26]

$$
\begin{aligned}
\hat{\sigma}_{A}(x)= & \frac{1}{16 \pi x^{2}}\left\{\sqrt{x} \sqrt{x-4}\left[96 g_{2}^{2} g_{Y}^{2}(x+4)+g_{Y}^{4}(65 x-68)+2 g_{2}^{4}(172+65 x)\right]\right. \\
& \left.-96\left[4 g_{2}^{2} g_{Y}^{2}(x-2)+g_{Y}^{4}(x-2)+4 g_{2}^{4}(x-1)\right] \ln \left(\frac{\sqrt{x-4} \sqrt{x}+x}{2}-1\right)\right\} .
\end{aligned}
$$

Taking into account the RGE of the gauge couplings at one loop, ${ }^{11}$ we obtain an accurate parametrization within $10 \%$ up to $z \lesssim 20$,

$$
\frac{\gamma_{A}}{s H z}=\frac{5.5035 \times 10^{15} \mathrm{GeV}}{g_{\star}^{3 / 2} M_{\mathcal{T}}} e^{-1.49 z^{1.0735}}
$$

Notice that under flavor rotations in Eq. (27), from Eqs. (56) and (68), we observe that

$$
f f^{\dagger} \rightarrow V^{*} f f^{\dagger} V^{T}, \quad \epsilon \rightarrow V^{*} \epsilon V^{T} .
$$

\footnotetext{
${ }^{11}$ The one-loop RGE equations of $\alpha_{2}=\frac{g_{2}^{2}}{4 \pi}$ and $\alpha_{Y}=\frac{g_{Y}^{2}}{4 \pi}$ are given by $[27,28], \quad \alpha_{2}(\mu)=\frac{12 \pi \alpha_{2}\left(m_{Z}\right)}{12 \pi-19 \alpha_{2}\left(m_{Z}\right)+19 \alpha_{2}\left(m_{Z}\right) \ln \mu}$, $\alpha_{Y}(\mu)=\frac{20 \pi \alpha_{Y}\left(m_{Z}\right)}{20 \pi+41 \alpha_{Y}\left(m_{Z}\right)-41 \alpha_{Y}\left(m_{Z}\right) \ln 2 \mu}$, respectively, where we take $\mu=$ $2 \pi T$ and fix $\alpha_{2}\left(m_{Z}\right)=0.0337$ and $\alpha_{Y}\left(m_{Z}\right)=0.0169$ with $m_{Z}=91.2 \mathrm{GeV}$.
}

For the source and washout terms (66) and (67) to transform the same way,

$$
S^{\mathrm{II}} \rightarrow V^{*} S^{\mathrm{II}} V^{T}, \quad W^{\mathrm{II}} \rightarrow V^{*} W^{\mathrm{II}} V^{T},
$$

one requires

$$
Y_{\Delta \ell} \rightarrow V^{*} Y_{\Delta \ell} V^{T}
$$

This can be obtained by a particular choice of ordering of flavor indices as discussed in Appendix A. Hence, we will take $y_{E} \rightarrow y_{E}^{*}$ in Eqs. (26) and (31) such that the transformation is consistent with the one above. Equivalently, we can take $Y_{\Delta \ell} \rightarrow Y_{\Delta \ell}^{T}$ in Eqs. (26) and (31).

For illustration, we choose a benchmark point from Ref. [22],

$$
m_{\mathcal{T}}=i m_{\nu}^{\mathrm{II}} \Rightarrow m_{\mathcal{H}}=(1-i) m_{\nu}^{\mathrm{II}},
$$



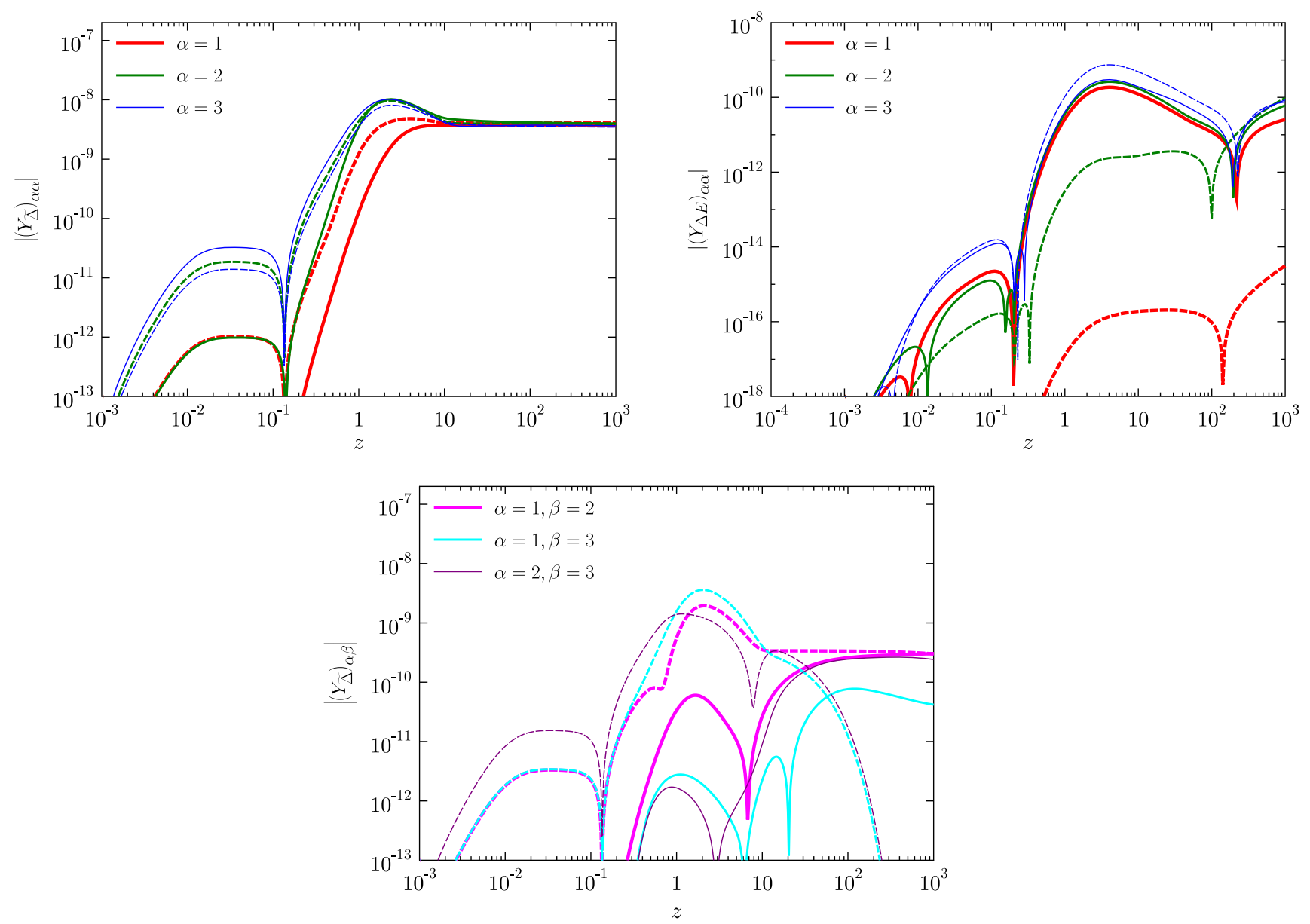

FIG. 3. Numerical solutions for type-II leptogenesis. In the top row, we plot the diagonal elements of $\left|Y_{\tilde{\Delta}}\right|$ and $\left|Y_{\Delta E}\right|$ in the two different bases: nonflavor basis $y_{E}$ (solid curves) and flavor basis $\hat{y}_{E}$ (dashed curves). In the bottom row, we plot the off-diagonal elements of $\left|Y_{\tilde{\Delta}}\right|$ and $\left|Y_{\Delta E}\right|$ in the $y_{E}$ (solid curves) and $\hat{y}_{E}$ (dashed curves) bases. Colors (thickness) denote different matrix elements as indicated in the plots. See the text for further discussions.

$$
\begin{gathered}
M_{\Delta}=5 \times 10^{12} \mathrm{GeV}, \\
|\mu|=0.1 M_{\mathcal{T}},
\end{gathered}
$$

and we fix the neutrino mass matrix to be

$$
m_{\nu}=r V_{E}^{T} U_{\mathrm{PMNS}}^{*} \operatorname{diag}\left(m_{1}, m_{2}, m_{3}\right) U_{\mathrm{PMNS}}^{\dagger} V_{E},
$$

with $m_{1}=10^{-3} \mathrm{eV}$, while for the rest of the parameters, we choose the best-fit parameters for normal mass ordering from the global fit [29]. The effect of RGE up to scale around $M_{\mathcal{T}}$ is accounted for approximately by taking $r=1.4$. We ignore the RGE of charge lepton Yukawa and fix it to be

$$
y_{E}=V_{E}^{\dagger} \hat{y}_{E} V_{E},
$$

where $\hat{y}_{E}=\operatorname{diag}\left(2.8 \times 10^{-6}, 5.9 \times 10^{-4}, 1.0 \times 10^{-2}\right)$. We will solve the Boltzmann equations in two different bases: nonflavor basis $y_{E}$ with $V_{E}=U_{\mathrm{PMNS}}^{\dagger}$ and flavor basis $\hat{y}_{E}$ with $V_{E}=I_{3 \times 3}$.

In Fig. 3, we show the numerical solutions comparing the results in $y_{E}$ (solid curves) and $\hat{y}_{E}$ (dashed curves) bases. In the top row, we show the diagonal elements of $\left|Y_{\tilde{\Delta}}\right|$ and $\left|Y_{\Delta E}\right|$, while in the bottom row, we show their off-diagonal elements. In the flavor basis $\hat{y}_{E}$, the off-diagonal elements start to become suppressed at various temperatures as the charged lepton Yukawa interactions subsequently get into thermal equilibrium. We see that at $z \sim 1000\left(Y_{\tilde{\Delta}}\right)_{12}$ and $\left(Y_{\Delta E}\right)_{12}$ remain large, indicating that one has not entered the three-flavor regime.

In Fig. 4, top row, we plot $\left|\operatorname{Tr} Y_{\tilde{\Delta}}\right|$ and $\left|\operatorname{Tr} Y_{\Delta E}\right|$ in the two bases: $y_{E}$ (red solid curve) and $\hat{y}_{E}$ (blue dashed curve). As expected, $\operatorname{Tr} Y_{\tilde{\Delta}}$ and $\operatorname{Tr} Y_{\Delta E}$ are basis independent, while the entries of $Y_{\tilde{\Delta}}$ and $Y_{\Delta E}$ differ among the two bases by the flavor rotations as in Eq. (28) with $V=U=V_{E}$. In the bottom plot of Fig. 4, we see that, while $\left|Y_{\Delta(B-L)}\right|=$ $\left|\operatorname{Tr} Y_{\tilde{\Delta}}-\operatorname{Tr} Y_{\Delta E}\right|$ is conserved at the end of leptogenesis, $z \gtrsim 20$, while $\left|\operatorname{Tr} Y_{\tilde{\Delta}}\right|$ and $\left|\operatorname{Tr} Y_{\Delta E}\right|$ continue to evolve. 

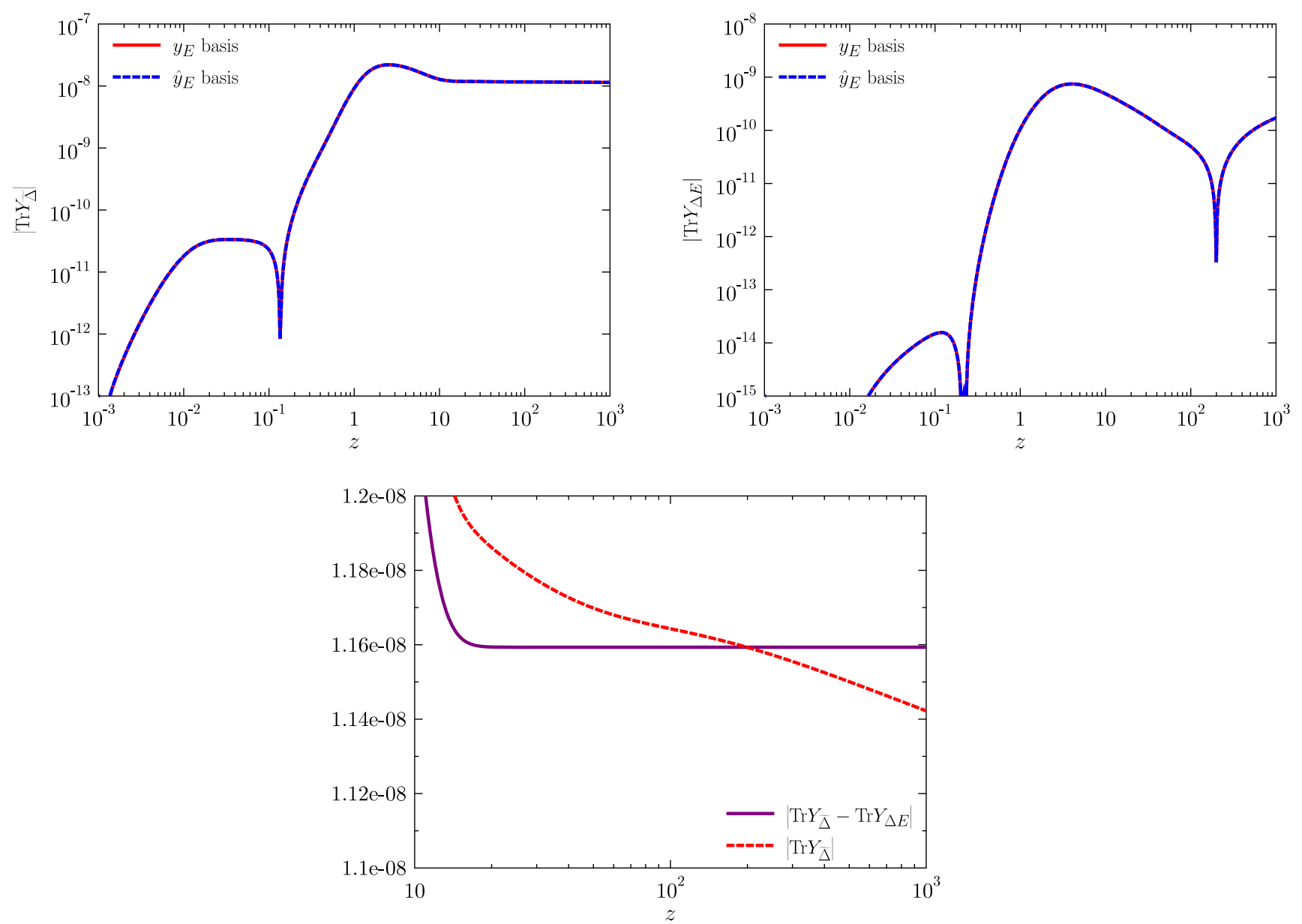

FIG. 4. Numerical solutions for type-II leptogenesis. In the top row, we plot $\left|\operatorname{Tr} Y_{\tilde{\Delta}}\right|$ and $\left|\operatorname{Tr} Y_{\Delta E}\right|$ in the two different bases: nonflavor basis $y_{E}$ (red solid curve) and flavor basis $\hat{y}_{E}$ (blue dashed curve). In the bottom plot, we plot $\left|\operatorname{Tr} Y_{\tilde{\Delta}}-\operatorname{Tr} Y_{\Delta E}\right|$ (purple solid curve) and $\left|\operatorname{Tr} Y_{\tilde{\Delta}}\right|$ (red dashed curve) for $z>10 .\left|\operatorname{Tr} Y_{\Delta E}\right|$ is too small to be shown here but one can easily deduced its value from the plot. See the text for further discussions.

In this example, the final baryon asymmetry obtained is $Y_{\Delta B}\left(T_{B-}\right)=3.6 \times 10^{-9}$.

\section{CONCLUSIONS}

In this work, we have developed a recipe to describe the evolution of lepton flavor charges from cosmic temperature ranging from $10^{15} \mathrm{GeV}$ down to the weak scale, taking into the full SM lepton flavor and spectator effects in a unified and lepton flavor basis-independent manner. This recipe can be applied to any leptogenesis model with the addition of arbitrary number of new scalars with nonzero hypercharges. We have shown that in order to describe leptogenesis in a basis-independent way and to take into account lepton flavor effect consistently it is necessary to describe both the charges of $\ell$ and $E$ in term of density matrices in their respective flavor spaces. To summarize, to apply this formalism to a leptogenesis model is to add the corresponding new physics interactions to the Boltzmann equations (26) and (31), and then the equations can be closed with Eqs. (41), (42), and those in Appendix C.
To demonstrate the applicability of this formalism, we have applied it to type-I and type-II leptogenesis models. Future direction will be to take into account baryon flavor effect.

\section{ACKNOWLEDGMENTS}

C.S.F. acknowledges the support by FAPESP Grant No. 2019/11197-6 for the project "Precision baryogenesis" and CNPq Grant No. 301271/2019-4. All the Boltzmann equations are solved with Mathematica sponsored by a colleague (who asked not to be named), while all the figures are prepared using Graphics Layout Engine [30]. A public code is currently under development.

\section{APPENDIX A: MATRIX OF NUMBER DENSITIES}

The density matrix operator of the SM in thermal equilibrium at temperature $T$ is given by

$$
\hat{\rho}_{\mathrm{SM}}=Z^{-1} e^{-\frac{1}{T}\left(\hat{H}_{\mathrm{SM}}-\sum_{i} \mu_{i} \hat{N}_{i}\right)},
$$


where $Z \equiv \operatorname{Tr}\left[e^{-\frac{1}{T}\left(\hat{H}_{\mathrm{SM}}-\sum_{i} \mu_{i} \hat{N}_{i}\right)}\right]$, with $\hat{H}_{\mathrm{SM}}$ the SM Hamiltonian and $\mu_{i}$ and $\hat{N}_{i}$ the chemical potential and number operator of a SM field $i$, respectively. If we are interested in the correlation between a particle species of different flavors, we can generalize the chemical potential and number operators to matrix in flavor space as

$\left(\mu_{i} \hat{N}_{i}\right)_{\alpha \beta} \equiv \int \frac{d^{3} p}{(2 \pi)^{3}}\left(\mu_{i}\right)_{\alpha \beta}\left(a_{i_{\beta}, \mathbf{p}}^{\dagger} a_{i_{\alpha}, \mathbf{p}}-b_{i_{\alpha}, \mathbf{p}}^{\dagger} b_{i_{\beta}, \mathbf{p}}\right)$,

no sum over $\alpha, \beta$,

where $\alpha$ and $\beta$ are flavor indices and we have made use of the fact that in chemical equilibrium with gauge bosons we have $\mu_{\bar{i}}=-\mu_{i}$. The operator $a_{i_{\alpha}, \mathbf{p}}^{\dagger}$ creates a particle $i_{\alpha}$ of momentum $\mathbf{p}$ from the vacuum $a_{i_{\alpha}, \mathbf{p}}^{\dagger}|0\rangle=\left|\mathbf{p}, i_{\alpha}\right\rangle$ while $b_{i_{\alpha}, \mathbf{p}}^{\dagger}$ creates an antilepton $\bar{i}_{\alpha}$ of momentum $\mathbf{p}$ as $b_{i_{\alpha}, \mathbf{p}}^{\dagger}|0\rangle=$ $\left|\mathbf{p}, \bar{i}_{\alpha}\right\rangle$. For fermions (bosons), they fulfill anticommutator (commutator) relations $\left[a_{i_{\beta}, \mathbf{p}^{\prime}}, a_{i_{\alpha}, \mathbf{p}}^{\dagger}\right]_{+(-)}=\left[b_{i_{\beta}, \mathbf{p}^{\prime}}, b_{i_{\alpha}, \mathbf{p}}^{\dagger}\right]_{+(-)}=$ $(2 \pi)^{3} \delta^{(3)}\left(\mathbf{p}-\mathbf{p}^{\prime}\right) \delta_{\alpha \beta}$, where we have defined $[A, B]_{+} \equiv$ $\{A, B\}=A B+B A \quad$ and $\quad[A, B]_{-} \equiv[A, B]=A B-B A$. Other operator combinations are zero. Sandwiching the operator (A2) between two states with particle of type $i$ of the same momentum $\mathbf{p}$ (but their flavors can be different), we have, for example, $\left\langle i_{\beta}, \mathbf{p}\left|\left(\mu_{i} \hat{N}_{i}\right)_{\alpha \beta}\right| i_{\alpha}, \mathbf{p}\right\rangle=\left(\mu_{i}\right)_{\alpha \beta}$. It also follows that $\left(\mu_{i}\right)_{\beta \alpha}=\left(\mu_{i}\right)_{\alpha \beta}^{*}$.

Next, we will define the generalized phase-space distribution $f_{i(\bar{i})}$ for particle $i$ and antiparticle $\bar{i}$, respectively, as [5]

$$
\begin{aligned}
& \delta_{\mathbf{p p}^{\prime}}\left(f_{i, \mathbf{p}}\right)_{\alpha \beta} \equiv \operatorname{Tr}\left[a_{i_{\beta}, \mathbf{p}}^{\dagger} a_{i_{\alpha}, \mathbf{p}^{\prime}} \hat{\rho}_{\mathrm{SM}}\right], \\
& \delta_{\mathbf{p p}^{\prime}}\left(f_{\bar{i}, \mathbf{p}}\right)_{\alpha \beta} \equiv \operatorname{Tr}\left[b_{i_{\alpha}, \mathbf{p}}^{\dagger} b_{i_{\beta}, \mathbf{p}^{\prime}} \hat{\rho}_{\mathrm{SM}}\right],
\end{aligned}
$$

where we have defined $\delta_{\mathbf{p p}^{\prime}} \equiv(2 \pi)^{3} \delta^{(3)}\left(\mathbf{p}-\mathbf{p}^{\prime}\right)$. Notice that the order of flavor indices in $f_{i(\bar{i}), \mathbf{p}}$ determines how it transforms under flavor rotation of the field. For instance, considering the fields to be $\ell_{\alpha}$ and $E_{\alpha}$, under unitary transformations in flavor space, $\ell \rightarrow V \ell$ and $E \rightarrow U E$, we have $f_{\ell(\bar{\ell}), \mathbf{p}} \rightarrow V f_{\ell(\bar{\ell}), \mathbf{p}} V^{\dagger}$ and $f_{E(\bar{E}), \mathbf{p}} \rightarrow U f_{E(\bar{E}), \mathbf{p}} U^{\dagger}$. If we have defined Eqs. (A3) and (A4) with $\left(f_{i, \mathbf{p}}\right)_{\beta \alpha}$ and $\left(f_{\bar{i}, \mathbf{p}}\right)_{\beta \alpha}$, they will transform as $f_{\ell(\bar{\ell}), \mathbf{p}} \rightarrow V^{*} f_{\ell(\bar{\ell}), \mathbf{p}} V^{T}$ and $f_{E(\bar{E}), \mathbf{p}} \rightarrow U^{*} f_{E(\bar{E}), \mathbf{p}} U^{T}$. Notice that $f_{i, \mathbf{p}}$ and $f_{\bar{i}, \mathbf{p}}$ are Hermitian.

In what follows, we would like to solve for $\left(f_{i, \mathbf{p}}\right)_{\alpha \beta}$ and $\left(f_{\bar{i}, \mathbf{p}}\right)_{\alpha \beta}$. Since the derivation below follows independently of whether $\mu_{i} \hat{N}_{i}$ is a matrix in flavor space or not, we will suppress the flavor indices. Notice that

$$
\begin{aligned}
Z & =\operatorname{Tr}\left[e^{-\frac{1}{T}\left(\hat{H}_{\mathrm{SM}}-\sum_{i} \mu_{i} \hat{N}_{i}\right)}\right] \\
& =\sum_{\text {states }}\left\langle\text { states }\left|e^{-\frac{1}{T}\left(\hat{H}_{\mathrm{SM}}-\sum_{i} \mu_{i} \hat{N}_{i}\right)}\right| \text { states }\right\rangle \\
& =\sum_{\text {states }}\left\langle\text { states }\left|e^{-\frac{1}{T} \sum_{i}\left(\mathcal{E}_{i}-\mu_{i}\right) \hat{N}_{i}}\right| \text { states }\right\rangle \\
& =\prod_{f}\left[1+e^{-\frac{1}{T}\left(\mathcal{E}_{f}-\mu_{f}\right)}\right] \prod_{b}\left[1-e^{-\frac{1}{T}\left(\mathcal{E}_{b}-\mu_{b}\right)}\right]^{-1} .
\end{aligned}
$$

The traces are taken over multiparticle states with energy $\mathcal{E}_{i}$. For fermion $f$, the occupation number is either 0 or 1 , and each of them contributes a factor of $1+e^{-\frac{1}{T}\left(\mathcal{E}_{f}-\mu_{f}\right)}$, while for boson $b$, each of them contributes a factor of $\sum_{n=0}^{\infty} e^{-\frac{1}{T}\left(\mathcal{E}_{b}-\mu_{b}\right) n}=\left[1-e^{-\frac{1}{T}\left(\mathcal{E}_{b}-\mu_{b}\right)}\right]^{-1}$.

For a fermion $i$, we have

$$
\begin{aligned}
\operatorname{Tr}\left[a_{i, \mathbf{p}}^{\dagger} a_{i, \mathbf{p}^{\mathbf{p}}} \hat{\rho}_{\mathrm{SM}}\right]= & Z^{-1} \delta_{\mathbf{p p}^{\prime}} e^{-\frac{1}{T}\left(\mathcal{E}_{i}-\mu_{i}\right)} \\
& \times \prod_{f \neq i}\left[1+e^{-\frac{1}{T}\left(\mathcal{E}_{f}-\mu_{f}\right)}\right] \prod_{b}\left[1-e^{-\frac{1}{T}\left(\mathcal{E}_{b}-\mu_{b}\right)}\right]^{-1} \\
= & \delta_{\mathbf{p p}^{\prime}} \frac{e^{-\frac{1}{T}\left(\mathcal{E}_{i}-\mu_{i}\right)}}{1+e^{-\frac{1}{T}\left(\mathcal{E}_{i}-\mu_{i}\right)}} \\
= & \delta_{\mathbf{p p}^{\prime}} \frac{1}{e^{\frac{1}{T}\left(\mathcal{E}_{i}-\mu_{i}\right)}+1} .
\end{aligned}
$$

For a boson $i$, we have

$$
\begin{aligned}
\operatorname{Tr}\left[a_{i, \mathbf{p}}^{\dagger} a_{i, \mathbf{p}^{\prime}} \hat{\rho}_{\mathrm{SM}}\right] & =Z^{-1} \delta_{\mathbf{p p}^{\prime}} \sum_{n} n e^{-\frac{1}{T}\left(\mathcal{E}_{i}-\mu_{i}\right) n} \prod_{f}\left[1+e^{-\frac{1}{T}\left(\mathcal{E}_{f}-\mu_{f}\right)}\right] \prod_{b \neq i}\left[1-e^{-\frac{1}{T}\left(\mathcal{E}_{b}-\mu_{b}\right)}\right]^{-1} \\
& =Z^{-1} \delta_{\mathbf{p p}^{\prime}} \frac{e^{-\frac{1}{T}\left(\mathcal{E}_{i}-\mu_{i}\right)}}{\left[1-e^{-\frac{1}{T}\left(\mathcal{E}_{i}-\mu_{i}\right)}\right]^{2}} \prod_{f}\left[1+e^{-\frac{1}{T}\left(\mathcal{E}_{f}-\mu_{f}\right)}\right] \prod_{b \neq i}\left[1-e^{-\frac{1}{T}\left(\mathcal{E}_{b}-\mu_{b}\right)}\right]^{-1} \\
& =\delta_{\mathbf{p p}^{\prime}} \frac{e^{-\frac{1}{T}\left(\mathcal{E}_{i}-\mu_{i}\right)}}{\left[1-e^{-\frac{1}{T}\left(\mathcal{E}_{i}-\mu_{i}\right)}\right]^{2}}\left[1-e^{-\frac{1}{T}\left(\mathcal{E}_{i}-\mu_{i}\right)}\right] \\
& =\delta_{\mathbf{p p}^{\prime}} \frac{1}{e^{\frac{1}{T}\left(\mathcal{E}_{i}-\mu_{i}\right)}-1} .
\end{aligned}
$$


In the second line above, we have used $\sum_{n} n e^{-\frac{1}{T}\left(\mathcal{E}_{i}-\mu_{i}\right) n}=-\frac{1}{\frac{1}{T}\left(\mathcal{E}_{i}-\mu_{i}\right)} \frac{d}{d n} \sum_{n} e^{-\frac{1}{T}\left(\mathcal{E}_{i}-\mu_{i}\right) n}=\frac{e^{-\frac{1}{T}\left(\mathcal{E}_{i}-\mu_{i}\right)}}{\left[1-e^{-\frac{1}{T}\left(\mathcal{E}_{i}-\mu_{i}\right)}\right]^{.}}$.

One can repeat the exercise above for antiparticle $\bar{i}$ with $a_{i} \rightarrow b_{i}$, and the only change is $\mu_{i} \rightarrow-\mu_{i}$. Hence, from the definitions (A3) and (A4), we obtain the desired results

$$
\left(f_{i, \mathbf{p}}\right)_{\alpha \beta}=\frac{1}{e^{\frac{\varepsilon_{i}-\left(\mu_{i}\right)_{\alpha \beta}}{T}}+\xi_{i}}, \quad\left(f_{\bar{i}, \mathbf{p}}\right)_{\alpha \beta}=\frac{1}{e^{\frac{\varepsilon_{i}+\left(\mu_{i}\right) \alpha \beta}{T}}+\xi_{i}},
$$

where $\xi_{i}=1(-1)$ for $i$ a fermion (boson) and $\mathcal{E}_{i}=\sqrt{|\mathbf{p}|^{2}+m_{i}^{2}}$. Integrating the phase space distributions above over 3-momentum, we obtain (matrices) of number densities

$$
\begin{aligned}
\left(n_{i}\right)_{\alpha \beta} & \equiv g_{i} \int \frac{d^{3} p}{(2 \pi)^{3}}\left(f_{i, \mathbf{p}}\right)_{\alpha \beta}, \\
\left(n_{\bar{i}}\right)_{\alpha \beta} & \equiv g_{i} \int \frac{d^{3} p}{(2 \pi)^{3}}\left(f_{\bar{i}, \mathbf{p}}\right)_{\alpha \beta},
\end{aligned}
$$

where we have included $g_{i}$ to take into account additional gauge degrees of freedom.

Expanding to linear order in chemical potential $\left|\mu_{i}\right| / T \ll 1$ and integrating over 3-momentum, the difference between the phase-space distributions of $i$ and $\bar{i}$, we obtain the (matrix of the) number density asymmetry

$$
\left(n_{\Delta i}\right)_{\alpha \beta} \equiv g_{i} \int \frac{d^{3} p}{(2 \pi)^{3}}\left[\left(f_{i, \mathbf{p}}\right)_{\alpha \beta}-\left(f_{\bar{i}, \mathbf{p}}\right)_{\alpha \beta}\right]=\frac{T^{2}}{6} g_{i} \zeta_{i}\left(\mu_{i}\right)_{\alpha \beta},
$$

where we have defined

$$
\zeta_{i} \equiv \frac{6}{\pi^{2}} \int_{m_{i} / T}^{\infty} d x x \sqrt{x^{2}-m_{i}^{2} / T^{2}} \frac{e^{x}}{\left(e^{x}+\xi_{i}\right)^{2}} .
$$

For massless particle $m_{i}=0$, we have $\zeta_{i}=1(2)$ for $i$ a massless fermion (boson). The transformation of $\left(n_{\Delta i}\right)_{\alpha \beta}$ follows directly from Eqs. (A3) and (A4). For instance, considering the fields to be $\ell_{\alpha}$ and $E_{\alpha}$, under unitary transformations in flavor space, $\ell \rightarrow V \ell$ and $E \rightarrow U E$, we have $n_{\Delta \ell} \rightarrow V n_{\Delta \ell} V^{\dagger}$ and $n_{\Delta E} \rightarrow U n_{\Delta E} U^{\dagger}$. Alternatively, if we have defined Eqs. (A3) and (A4) with $\left(f_{i, \mathbf{p}}\right)_{\beta \alpha}$ and $\left(f_{\bar{i}, \mathbf{p}}\right)_{\beta \alpha}$, the transformations will be $n_{\Delta \ell} \rightarrow V^{*} n_{\Delta \ell} V^{T}$ and $n_{\Delta E} \rightarrow U^{*} n_{\Delta E} U^{T}$.

Normalizing Eq. (A10) by the cosmic entropy density $s=\frac{2 \pi^{2}}{45} g_{\star} T^{3}$ with $g_{\star}$ being the effective relativistic degrees of freedom of the Universe, we have

$$
\left(Y_{\Delta i}\right)_{\alpha \beta} \equiv \frac{\left(n_{\Delta i}\right)_{\alpha \beta}}{s} \equiv Y^{\mathrm{nor}} g_{i} \zeta_{i} \frac{2\left(\mu_{i}\right)_{\alpha \beta}}{T}
$$

where we have defined $Y^{\text {nor }} \equiv \frac{15}{8 \pi^{2} g_{\star}}$. The relation above also holds for a particle which does not carry a flavor index, e.g., for the SM Higgs, which is taken to be massless at high temperature, we have $Y_{\Delta H}=4 Y^{\text {nor }} \frac{2 \mu_{H}}{T}$, where $g_{H}=2$ for the $S U(2)_{L}$ gauge degrees of freedom and $\xi_{H}=2$ for massless boson.

\section{APPENDIX B: COVARIANT FLAVOR STRUCTURES OF KINETIC EQUATIONS}

The complete flavor-covariant kinetic equations have been derived in Refs. [6,7] using the closed time path formalism. Here, we would like to sketch how the same flavor structures of the kinetic equations for $\ell$ and $E$ arise by considering the evolution equation of a Heisenberg operator [5].

We will start by deriving some relations relating the equilibrium phase-space distributions with the (matrices) of number density asymmetries. As shown the previous section, for a particle $i$ which is in kinetic equilibrium, its phase-space distribution is given by Eq. (A8). For a process $a b \ldots \leftrightarrow i j \ldots$ which is in chemical equilibrium $\mu_{a}+\mu_{b}+\cdots=\mu_{i}+\mu_{j}+\ldots$ [if a particle carries a family index, e.g., $i_{\alpha}$, the chemical potential refers to the corresponding diagonal element $\left(\mu_{i}\right)_{\alpha \alpha}$ ], we can verify that the following identity is satisfied,

$$
\begin{aligned}
& f_{a} f_{b} \ldots\left(1-\xi_{i} f_{i}\right)\left(1-\xi_{j} f_{j}\right) \ldots \\
& \quad=f_{i} f_{j} \ldots\left(1-\xi_{a} f_{a}\right)\left(1-\xi_{b} f_{b}\right) \ldots
\end{aligned}
$$

where we have used energy conservation $\mathcal{E}_{a}+\mathcal{E}_{b}+\cdots=$ $\mathcal{E}_{i}+\mathcal{E}_{j}+\ldots$ and we have suppressed the subscript of momentum/energy in the distribution functions. In general, we cannot make use of the identity above since chemical equilibrium condition is not necessarily fulfilled when the corresponding process is slower than the Hubble expansion rate. Defining the distribution of a particle $i$ in kinetic equilibrium with zero chemical potential as $f_{i}^{\mathrm{eq}}$, the following identity is clearly satisfied:

$$
\begin{aligned}
& f_{a}^{\mathrm{eq}} f_{b}^{\mathrm{eq}} \ldots\left(1-\xi_{i} f_{i}^{\mathrm{eq}}\right)\left(1-\xi_{j} f_{j}^{\mathrm{eq}}\right) \ldots \\
& \quad=f_{i}^{\mathrm{eq}} f_{j}^{\mathrm{eq}} \ldots\left(1-\xi_{a} f_{a}^{\mathrm{eq}}\right)\left(1-\xi_{b} f_{b}^{\mathrm{eq}}\right) \ldots
\end{aligned}
$$

In the following, let us consider all the particles are in kinetic equilibrium (this holds for all the SM particles which experience gauge interactions). Expanding in chemical potentials $\left|\mu_{i}\right| / T \ll 1$ up to linear order for all the particles, we have 


$$
\begin{aligned}
& \frac{f_{a} f_{b} \ldots\left(1-\xi_{i} f_{i}\right)\left(1-\xi_{j} f_{j}\right) \ldots}{f_{a}^{\mathrm{eq}} f_{b}^{\mathrm{eq}} \ldots\left(1-\xi_{i} f_{i}^{\mathrm{eq}}\right)\left(1-\xi_{j} f_{j}^{\mathrm{eq}}\right) \ldots} \\
& =\left[1+\frac{\mu_{a}}{T}\left(1-\xi_{a} f_{a}^{\mathrm{eq}}\right)+\frac{\mu_{b}}{T}\left(1-\xi_{b} f_{b}^{\mathrm{eq}}\right)+\cdots\right] \\
& \quad \times\left(1-\frac{\mu_{i}}{T} \xi_{i} f_{i}^{\mathrm{eq}}-\frac{\mu_{j}}{T} \xi_{j} f_{j}^{\mathrm{eq}}+\cdots\right) \\
& =1+\sum_{I=a, b, \ldots} \frac{\mu_{I}}{T}-\sum_{I=a, b, \ldots} \frac{\mu_{I}}{T} \xi_{I} f_{I}^{\mathrm{eq}}-\sum_{F=i, j, \ldots} \frac{\mu_{F}}{T} \xi_{F} f_{F}^{\mathrm{eq}} \\
& =1+\sum_{I=a, b, \ldots} \frac{Y_{\Delta I}}{2 Y^{\mathrm{nor}} g_{I} \zeta_{I}}-\sum_{A=a, b, \ldots, i, j, \ldots} \frac{Y_{\Delta A} \xi_{A} f_{A}^{\mathrm{eq}}}{2 Y_{A}^{\mathrm{nor}} g_{A}}
\end{aligned}
$$

where in the last step we have used Eq. (A12). Similarly, we have the relation for antiparticles by changing the sign of the chemical potentials,

$$
\begin{aligned}
& \frac{f_{\bar{a}} f_{\bar{b}} \ldots\left(1-\xi_{i} f_{\bar{i}}\right)\left(1-\xi_{j} f_{\bar{j}}\right) \ldots}{f_{a}^{\mathrm{eq}} f_{b}^{\mathrm{eq}} \ldots\left(1-\xi_{i} f_{i}^{\mathrm{eq}}\right)\left(1-\xi_{j} f_{j}^{\mathrm{eq}}\right) \ldots} \\
& \quad=1-\sum_{I=a, b, \ldots} \frac{Y_{\Delta I}}{2 Y^{\mathrm{nor}} g_{I} \zeta_{I}}+\sum_{A=a, b, \ldots, i, j, \ldots} \frac{Y_{\Delta A} \xi_{A} f_{A}^{\mathrm{eq}}}{2 Y^{\mathrm{nor}} g_{A} \zeta_{A}}
\end{aligned}
$$

The evolution equations of the Heisenberg operators $\left(\mathcal{O}_{i, \mathbf{p}}\right)_{\alpha \beta}(t) \equiv a_{i_{\beta}, \mathbf{p}}^{\dagger}(t) a_{i_{\alpha}, \mathbf{p}}(t) \quad$ and $\quad\left(\mathcal{O}_{\bar{i}, \mathbf{p}}\right)_{\alpha \beta}(t) \equiv$ $b_{i_{\alpha}, \mathbf{p}}^{\dagger}(t) b_{i_{\beta}, \mathbf{p}}(t)$ are given by

$\frac{\partial\left(\mathcal{O}_{i, \mathbf{p}}\right)_{\alpha \beta}}{\partial t}=i\left[\hat{H},\left(\mathcal{O}_{i, \mathbf{p}}\right)_{\alpha \beta}\right], \quad \frac{\partial\left(\mathcal{O}_{\bar{i}, \mathbf{p}}\right)_{\alpha \beta}}{\partial t}=i\left[\hat{H},\left(\mathcal{O}_{\bar{i}, \mathbf{p}}\right)_{\alpha \beta}\right]$,

where $\hat{H}=\hat{H}_{0}+\hat{H}_{\text {int }}$ is the Hamiltonian of the system with $H_{0}$ denoting the free field Hamiltonian, while $\hat{H}_{\text {int }}$ represents all possible interactions among the fields. In the following, we will write down the derivation only for the equation of motion of $\mathcal{O}_{i, \mathbf{p}}$ since those for $\mathcal{O}_{\bar{i}, \mathbf{p}}$ will be analogous.

Taking the ensemble average on both sides of Eq. (B5), we have

$$
\frac{\partial\left(f_{i, \mathbf{p}}\right)_{\alpha \beta}}{\partial t}=i\left\langle\left[\hat{H},\left(\mathcal{O}_{i, \mathbf{p}}\right)_{\alpha \beta}\right]\right\rangle
$$

where we have denoted $\langle\mathcal{O}\rangle \equiv \operatorname{Tr}\left[\mathcal{O} \hat{\rho}_{\mathrm{SM}}\right]$. The effect of cosmic expansion can be taken into account by adding the following:

$$
\frac{\partial\left(f_{i, \mathbf{p}}\right)_{\alpha \beta}}{\partial t}-\mathcal{H}|\mathbf{p}| \frac{\partial\left(f_{i, \mathbf{p}}\right)_{\alpha \beta}}{\partial|\mathbf{p}|}=i\left\langle\left[\hat{H},\left(\mathcal{O}_{i, \mathbf{p}}\right)_{\alpha \beta}\right]\right\rangle
$$

Integrating the equation above over momentum $\mathbf{p}$ on both sides, we have

$$
\frac{d\left(n_{i}\right)_{\alpha \beta}}{d t}+3 \mathcal{H}\left(n_{i}\right)_{\alpha \beta}=i \int \frac{d^{3} p}{(2 \pi)^{3}}\left\langle\left[\hat{H},\left(\mathcal{O}_{i, \mathbf{p}}\right)_{\alpha \beta}\right]\right\rangle,
$$

where we have defined the number density (matrix) as

$$
\left(n_{i}\right)_{\alpha \beta} \equiv \int \frac{d^{3} p}{(2 \pi)^{3}}\left(f_{i, \mathbf{p}}\right)_{\alpha \beta},
$$

and assume that $f_{i, \mathbf{p}}$ goes to zero at large momentum. In the absence of interactions $\hat{H}_{\text {int }}=0$, the phase space will evolve purely due to the Hubble expansion. In terms of $Y_{i} \equiv n_{i} / s$, we can rewrite

$$
\frac{d\left(n_{i}\right)_{\alpha \beta}}{d t}+3 \mathcal{H}\left(n_{i}\right)_{\alpha \beta}=s \frac{d\left(Y_{i}\right)_{\alpha \beta}}{d t}
$$

For massless fields, $H_{0}$ does not contribution to the righthand side of Eq. (B8). Next, we would like to write the terms in right-hand side of evolution equation also in terms of number densities. Doing a perturbative expansion on the Heisenberg operator $\left[\hat{H}_{\text {int }},\left(\mathcal{O}_{i, \mathbf{p}}\right)_{\alpha \beta}\right]$ to the first order in $\hat{H}_{\text {int }}$, and considering that the interaction timescale is much shorter than the evolution timescale, we can take the time integral to infinity and obtain [5]

$$
\begin{aligned}
s & \frac{d\left(Y_{i}\right)_{\alpha \beta}}{d t} \\
= & i \int \frac{d^{3} p}{(2 \pi)^{3}}\left\langle\left[\hat{H}_{\mathrm{int}, 0}(0),\left(\mathcal{O}_{i, \mathbf{p}}\right)_{\alpha \beta, 0}(0)\right]\right\rangle \\
& -\int \frac{d^{3} p}{(2 \pi)^{3}} \int_{0}^{\infty} d t\left\langle\left[\hat{H}_{\mathrm{int}, 0}(t),\left[\hat{H}_{\mathrm{int}, 0}(0),\left(\mathcal{O}_{i, \mathbf{p}}\right)_{\alpha \beta, 0}(0)\right]\right]\right\rangle,
\end{aligned}
$$

where the subscript 0 denote operators consist of free fields, i.e., $\hat{H}_{\text {int }}=0$.

Considering only the SM charged lepton Yukawa interaction term, we have

$$
\hat{H}_{\mathrm{int}}=\int d^{3} x\left[\left(y_{E}\right)_{\alpha \beta} \overline{E_{\alpha}} \ell_{\beta} H^{*}+\left(y_{E}\right)_{\alpha \beta}^{*} \overline{\ell_{\beta}} E_{\alpha} H\right] .
$$

Since this interaction is linear in the each type of field, it will only contribute to the second term. Considering thermal mass [31], there is a contribution to the first term of Eq. (B11), which results in oscillation among $\ell$ flavors. Reference [6] showed that flavor oscillations are damped by gauge interactions, and hence we will ignore this term.

Expanding the fields in momentum modes, we have

$$
\ell_{\beta}=\int \frac{d^{3} p}{(2 \pi)^{3} \sqrt{2 \mathcal{E}}} \sum_{s}\left(a_{\ell_{\beta}, \mathbf{p}}^{(s)} u_{\ell, \mathbf{p}}^{(s)} e^{-i p \cdot x}+b_{\ell_{\beta}, \mathbf{p}}^{(s) \dagger} v_{\ell, \mathbf{p}}^{(s)} e^{+i p \cdot x}\right),
$$




$$
\begin{gathered}
E_{\alpha}=\int \frac{d^{3} p}{(2 \pi)^{3} \sqrt{2 \mathcal{E}}} \sum_{s}\left(a_{E_{\alpha}, \mathbf{p}}^{(s)} u_{E, \mathbf{p}}^{(s)} e^{-i p \cdot x}+b_{E_{\alpha}, \mathbf{p}}^{(s) \dagger} v_{E, \mathbf{p}}^{(s)} e^{+i p \cdot x}\right), \\
H=\int \frac{d^{3} p}{(2 \pi)^{3} \sqrt{2 \mathcal{E}}}\left(a_{H, \mathbf{p}} e^{-i p \cdot x}+b_{H, \mathbf{p}}^{\dagger} e^{+i p \cdot x}\right),
\end{gathered}
$$

where $p \cdot x=\mathcal{E} t-\mathbf{p} \cdot \mathbf{x}$ and the sum $s$ is taken over the two spin states. Substituting the fields above into Eq. (B11), we obtain the evolution equation of $\ell$ as

$$
\begin{aligned}
s \frac{d\left(Y_{\ell}\right)_{\alpha \beta}}{d t}= & -\frac{1}{2} \int \frac{d^{3} p}{(2 \pi)^{3} 2 \mathcal{E}_{\ell}} \frac{d^{3} p_{E}}{(2 \pi)^{3} 2 \mathcal{E}_{E}} \frac{d^{3} p_{H}}{(2 \pi)^{3} 2 \mathcal{E}_{H}} 2 p \cdot p_{E} \\
& \times\left\{\delta_{p_{E}-p_{\ell}+p_{H}}\left[\left\{f_{\ell, \mathbf{p}}, y_{E}^{\dagger}\left(I-f_{E, \mathbf{p}_{E}}\right) y_{E}\right\}_{\alpha \beta}\left(1+f_{H, \mathbf{p}_{H}}\right)-\left\{\left(I-f_{\ell, \mathbf{p}}\right), y_{E}^{\dagger} f_{E, \mathbf{p}_{E}} y_{E}\right\}_{\alpha \beta} f_{H, \mathbf{p}_{H}}\right]\right. \\
& +\delta_{p_{E}-p_{\ell}-p_{H}}\left[\left\{f_{\ell, \mathbf{p}}, y_{E}^{\dagger}\left(I-f_{E, \mathbf{p}_{E}}\right) y_{E}\right\}_{\alpha \beta} f_{\bar{H}, \mathbf{p}_{H}}-\left\{\left(I-f_{\ell, \mathbf{p}}\right), y_{E}^{\dagger} f_{E, \mathbf{p}_{E}} y_{E}\right\}_{\alpha \beta}\left(1+f_{\bar{H}, \mathbf{p}_{H}}\right)\right] \\
& +\delta_{p_{E}+p_{\ell}-p_{H}}\left[\left\{f_{\ell, \mathbf{p}}, y_{E}^{\dagger} f_{\bar{E}, \mathbf{p}_{E}} y_{E}\right\}_{\alpha \beta}\left(1+f_{H, \mathbf{p}_{H}}\right)-\left\{\left(I-f_{\ell, \mathbf{p}}\right), y_{E}^{\dagger}\left(I-f_{\bar{E}, \mathbf{p}_{E}}\right) y_{E}\right\}_{\alpha \beta} f_{H, \mathbf{p}_{H}}\right] \\
& \left.+\delta_{p_{E}+p_{\ell}+p_{H}}\left[\left\{f_{\ell, \mathbf{p}}, y_{E}^{\dagger} f_{\bar{E}, \mathbf{p}_{E}} y_{E}\right\}_{\alpha \beta} f_{\bar{H}, \mathbf{p}_{H}}-\left\{\left(I-f_{\ell, \mathbf{p}}\right), y_{E}^{\dagger}\left(I-f_{\bar{E}, \mathbf{p}_{E}}\right) y_{E}\right\}_{\alpha \beta}\left(1+f_{\bar{H}, \mathbf{p}_{H}}\right)\right]\right\},
\end{aligned}
$$

where $\delta_{p} \equiv(2 \pi)^{4} \delta^{(4)}(p)$ with $p$ a 4-momentum, $I$ is a $3 \times 3$ identity matrix, and we have assumed all external fields to be massless. Clearly, the whole term vanishes since $p \cdot p_{E} \propto p_{H}^{2}=0$.

For nonvanishing result, one should consider thermal masses and scattering processes involving another external field [20]. For instance, a gauge field can be attached to either $\ell, E$, or $H$. One can also attach a fermion-antifermion pair to the Higgs fields, and the process involving the topquark Yukawa coupling will be the dominant one. Since the flavor structures involving $\ell$ and $E$ will remain exactly the same, we will not carry out the exercise here. ${ }^{12}$ Notice that the last term in the big curly brackets of Eq. (B16) will still be zero due to energy-momentum conservation.

Dividing and multiplying the terms in the right-hand side of Eq. (B16) by $f_{\ell, \mathbf{p}}^{\mathrm{eq}}\left(1-f_{E, \mathbf{p}_{E}}^{\mathrm{eq}}\right)\left(1+f_{H, \mathbf{p}_{H}}^{\mathrm{eq}}\right)=$ $f_{E, \mathbf{p}_{E}}^{\mathrm{eq}} f_{H, \mathbf{p}_{H}}^{\mathrm{eq}}\left(1-f_{\ell, \mathbf{p}}^{\mathrm{eq}}\right)$, which follows from Eq. (B2) and expanding up to linear term in $\left|\mu_{i}\right| / T \ll 1$, the first three terms in the big curly brackets in Eq. (B16) all have the same flavor structure,

$$
\begin{aligned}
s \frac{d Y_{\ell}}{d t} \sim & -\frac{1}{2 Y^{\text {nor }}}\left[\left\{y_{E}^{\dagger} y_{E}, \frac{Y_{\Delta \ell}}{g_{\ell} \zeta_{\ell}}\right\}-2\left(\frac{y_{E}^{\dagger} Y_{\Delta E} y_{E}}{g_{E} \zeta_{E}}\right)\right. \\
& \left.-2\left(y_{E}^{\dagger} y_{E}\right) \frac{Y_{\Delta H}}{g_{H} \zeta_{H}}\right],
\end{aligned}
$$

where we have made used of Eq. (B3). Similarly, we obtain the evolution equation of $\bar{l}$ by changing the sign of all chemical potentials,

\footnotetext{
${ }^{12}$ For the scatterings involving two quarks instead of the Higgs fields, one can still rewrite in terms of Higgs number density asymmetry for $T<T_{t} \sim 10^{15} \mathrm{GeV}$ when interactions involving the top-quark Yukawa are in equilibrium.
}

$$
\begin{aligned}
s \frac{d Y_{\bar{\ell}}}{d t} \sim & \frac{1}{2 Y^{\text {nor }}}\left[\left\{y_{E}^{\dagger} y_{E}, \frac{Y_{\Delta \ell}}{g_{\ell} \zeta_{\ell}}\right\}-2\left(\frac{y_{E}^{\dagger} Y_{\Delta E} y_{E}}{g_{E} \zeta_{E}}\right)\right. \\
& \left.-2\left(y_{E}^{\dagger} y_{E}\right) \frac{Y_{\Delta H}}{g_{H} \zeta_{H}}\right] .
\end{aligned}
$$

Hence, the evolution equation for $Y_{\Delta \ell}=Y_{\ell}-Y_{\bar{\ell}}$ has the following form:

$$
\begin{aligned}
s \frac{d Y_{\Delta \ell}}{d t} \sim & -\frac{1}{Y^{\text {nor }}}\left[\left\{y_{E}^{\dagger} y_{E}, \frac{Y_{\Delta \ell}}{g_{\ell} \zeta_{\ell}}\right\}\right. \\
& \left.-2 \frac{y_{E}^{\dagger} Y_{\Delta E} y_{E}}{g_{E} \zeta_{E}}-2 y_{E}^{\dagger} y_{E} \frac{Y_{\Delta H}}{g_{H} \zeta_{H}}\right] .
\end{aligned}
$$

Repeating the exercise above, we obtain the evolution equation for $Y_{\Delta E}=Y_{E}-Y_{\bar{E}}$ with the following flavor structure:

$$
\begin{aligned}
s \frac{d Y_{\Delta E}}{d t} \sim & -\frac{1}{Y^{\text {nor }}}\left[\left\{y_{E} y_{E}^{\dagger}, \frac{Y_{\Delta E}}{g_{E} \zeta_{E}}\right\}-2 \frac{y_{E} Y_{\Delta \ell} y_{E}^{\dagger}}{g_{\ell} \zeta_{\ell}}\right. \\
& \left.+2 y_{E} y_{E}^{\dagger} \frac{Y_{\Delta H}}{g_{H} \zeta_{H}}\right] .
\end{aligned}
$$

Under rotations in flavor spaces $E \rightarrow U E, \ell \rightarrow V \ell$, $y_{E} \rightarrow U y_{E} V^{\dagger}$, the kinetic equations above will have the same form (flavor covariant) since $Y_{\Delta \ell} \rightarrow V Y_{\Delta \ell} V^{\dagger}$ and $Y_{\Delta E} \rightarrow U Y_{\Delta E} U^{\dagger}$.

As a final remark, in a radiation-dominated Universe and assuming entropy conservation, we can trade the time variable with temperature $T$ using the relation

$$
\frac{d z}{d t}=z \mathcal{H}
$$


where we have defined $z \equiv \frac{M_{\text {ref }}}{T}$ with $M_{\text {ref }}$ an arbitrary mass scale.

\section{APPENDIX C: QUARK NUMBER ASYMMETRIES}

Here, we list the relations between quark number asymmetries with $Y_{\tilde{\Delta}}$ and $Y_{\Delta E}$ assuming all other conserved charges are zero. We have included the contributions from possible new scalar fields $\phi_{i}$ with hypercharge $q_{\phi_{i}}^{Y}$. For $T>T_{u}$, all quark number asymmetries are independent of $Y_{\tilde{\Delta}}, Y_{\Delta E}$, and $Y_{\Delta \phi_{i}}$.

For $T_{u}<T<T_{t}$, we have

$$
\begin{aligned}
& Y_{\Delta Q_{3}}=\frac{1}{3}\left(\operatorname{Tr} Y_{\tilde{\Delta}}-2 \operatorname{Tr} Y_{\Delta E}+2 \sum_{i} q_{\phi_{i}}^{Y} Y_{\Delta \phi_{i}}\right), \\
& Y_{\Delta t}=-\frac{1}{3}\left(\operatorname{Tr} Y_{\tilde{\Delta}}-2 \operatorname{Tr} Y_{\Delta E}+2 \sum_{i} q_{\phi_{i}}^{Y} Y_{\Delta \phi_{i}}\right),
\end{aligned}
$$

while the rest are independent of $Y_{\tilde{\Delta}}, Y_{\Delta E}$, and $Y_{\Delta \phi_{i}}$.

For $T_{B}<T<T_{u}$, we have

$$
\begin{array}{cr}
Y_{\Delta Q_{1}}=Y_{\Delta Q_{2}}=-\frac{3}{23}\left(\operatorname{Tr} Y_{\tilde{\Delta}}-2 \operatorname{Tr} Y_{\Delta E}+2 \sum_{i} q_{\phi_{i}}^{Y} Y_{\Delta \phi_{i}}\right), & (\mathrm{C} 3) \\
Y_{\Delta Q_{3}}=\frac{6}{23}\left(\operatorname{Tr} Y_{\tilde{\Delta}}-2 \operatorname{Tr} Y_{\Delta E}+2 \sum_{i} q_{\phi_{i}}^{Y} Y_{\Delta \phi_{i}}\right), & (\mathrm{C} 4) \\
Y_{\Delta u}=Y_{\Delta d}=Y_{\Delta c}=Y_{\Delta s}=Y_{\Delta b} & \\
= & \frac{3}{46}\left(\operatorname{Tr} Y_{\tilde{\Delta}}-2 \operatorname{Tr} Y_{\Delta E}+2 \sum_{i} q_{\phi_{i}}^{Y} Y_{\Delta \phi_{i}}\right), \\
Y_{\Delta t}=-\frac{15}{46}\left(\operatorname{Tr} Y_{\tilde{\Delta}}-2 \operatorname{Tr} Y_{\Delta E}+2 \sum_{i} q_{\phi_{i}}^{Y} Y_{\Delta \phi_{i}}\right) . & (\mathrm{C} 6)
\end{array}
$$

For $T_{u-b}<T<T_{B}$, we have

$$
Y_{\Delta Q_{1}}=Y_{\Delta Q_{2}}=\frac{2}{115}\left(4 \operatorname{Tr} Y_{\tilde{\Delta}}+15 \operatorname{Tr} Y_{\Delta E}-15 \sum_{i} q_{\phi_{i}}^{Y} Y_{\Delta \phi_{i}}\right)
$$

$$
Y_{\Delta Q_{3}}=\frac{1}{115}\left(53 \operatorname{Tr} Y_{\tilde{\Delta}}-60 \operatorname{Tr} Y_{\Delta E}+60 \sum_{i} q_{\phi_{i}}^{Y} Y_{\Delta \phi_{i}}\right),
$$

$$
\begin{aligned}
Y_{\Delta u} & =Y_{\Delta d}=Y_{\Delta c}=Y_{\Delta s}=Y_{\Delta b} \\
& =\frac{1}{115}\left(19 \operatorname{Tr} Y_{\tilde{\Delta}}-15 \operatorname{Tr} Y_{\Delta E}+15 \sum_{i} q_{\phi_{i}}^{Y} Y_{\Delta \phi_{i}}\right),
\end{aligned}
$$

$$
Y_{\Delta t}=-\frac{1}{115}\left(26 \operatorname{Tr} Y_{\tilde{\Delta}}-75 \operatorname{Tr} Y_{\Delta E}+75 \sum_{i} q_{\phi_{i}}^{Y} Y_{\Delta \phi_{i}}\right) .
$$

For $T_{u-c}<T<T_{u-b}$, we have

$$
\begin{gathered}
Y_{\Delta Q_{1}}=Y_{\Delta Q_{2}}=Y_{\Delta Q_{3}}=\frac{1}{5} \operatorname{Tr} Y_{\tilde{\Delta}}, \\
Y_{\Delta u}=Y_{\Delta d}=Y_{\Delta c}=Y_{\Delta s}=\frac{1}{10} \operatorname{Tr} Y_{\tilde{\Delta}} \\
Y_{\Delta t}=-\frac{1}{5}\left(\operatorname{Tr} Y_{\tilde{\Delta}}-3 \operatorname{Tr} Y_{\Delta E}+3 \sum_{i} q_{\phi_{i}}^{Y} Y_{\Delta \phi_{i}}\right), \\
Y_{\Delta b}=\frac{1}{5}\left(2 \operatorname{Tr} Y_{\tilde{\Delta}}-3 \operatorname{Tr} Y_{\Delta E}+3 \sum_{i} q_{\phi_{i}}^{Y} Y_{\Delta \phi_{i}}\right) .
\end{gathered}
$$

For $T_{B_{3}-B_{2}}<T<T_{u-c}$, we have

$$
Y_{\Delta Q_{1}}=\frac{1}{130}\left(11 \operatorname{Tr} Y_{\tilde{\Delta}}+30 \operatorname{Tr} Y_{\Delta E}-30 \sum_{i} q_{\phi_{i}}^{Y} Y_{\Delta \phi_{i}}\right),
$$

$$
Y_{\Delta Q_{2}}=\frac{1}{130}\left(41 \operatorname{Tr} Y_{\tilde{\Delta}}-30 \operatorname{Tr} Y_{\Delta E}+30 \sum_{i} q_{\phi_{i}}^{Y} Y_{\Delta \phi_{i}}\right),
$$

$$
\begin{aligned}
Y_{\Delta u} & =Y_{\Delta d}=Y_{\Delta s} \\
& =\frac{1}{260}\left(41 \operatorname{Tr} Y_{\tilde{\Delta}}-30 \operatorname{Tr} Y_{\Delta E}+30 \sum_{i} q_{\phi_{i}}^{Y} Y_{\Delta \phi_{i}}\right),
\end{aligned}
$$

$$
Y_{\Delta c}=-\frac{1}{260}\left(19 \operatorname{Tr} Y_{\tilde{\Delta}}-90 \operatorname{Tr} Y_{\Delta E}+90 \sum_{i} q_{\phi_{i}}^{Y} Y_{\Delta \phi_{i}}\right),
$$

$$
Y_{\Delta t}=-\frac{1}{130}\left(17 \operatorname{Tr} Y_{\tilde{\Delta}}-60 \operatorname{Tr} Y_{\Delta E}+60 \sum_{i} q_{\phi_{i}}^{Y} Y_{\Delta \phi_{i}}\right)
$$

$$
Y_{\Delta b}=\frac{1}{130}\left(43 \operatorname{Tr} Y_{\tilde{\Delta}}-60 \operatorname{Tr} Y_{\Delta E}+60 \sum_{i} q_{\phi_{i}}^{Y} Y_{\Delta \phi_{i}}\right)
$$


For $T_{u-s}<T<T_{B_{3}-B_{2}}$, we have

$$
\begin{gathered}
Y_{\Delta Q_{1}}=\frac{1}{10}\left(\operatorname{Tr} Y_{\tilde{\Delta}}+2 \operatorname{Tr} Y_{\Delta E}-2 \sum_{i} q_{\phi_{i}}^{Y} Y_{\Delta \phi_{i}}\right), \quad(\mathrm{C} 22) \\
Y_{\Delta Q_{2}}=Y_{\Delta Q_{3}}=\frac{1}{20}\left(5 \operatorname{Tr} Y_{\tilde{\Delta}}-2 \operatorname{Tr} Y_{\Delta E}+2 \sum_{i} q_{\phi_{i}}^{Y} Y_{\Delta \phi_{i}}\right),
\end{gathered}
$$

$Y_{\Delta u}=Y_{\Delta d}=Y_{\Delta s}=\frac{1}{20}\left(3 \operatorname{Tr} Y_{\tilde{\Delta}}-2 \operatorname{Tr} Y_{\Delta E}+2 \sum_{i} q_{\phi_{i}}^{Y} Y_{\Delta \phi_{i}}\right)$,

$Y_{\Delta c}=Y_{\Delta t}=-\frac{1}{10}\left(\operatorname{Tr} Y_{\tilde{\Delta}}-4 \operatorname{Tr} Y_{\Delta E}+4 \sum_{i} q_{\phi_{i}}^{Y} Y_{\Delta \phi_{i}}\right)$,

$Y_{\Delta b}=\frac{1}{20}\left(7 \operatorname{Tr} Y_{\tilde{\Delta}}-10 \operatorname{Tr} Y_{\Delta E}+10 \sum_{i} q_{\phi_{i}}^{Y} Y_{\Delta \phi_{i}}\right)$.

For $T_{u-d}<T<T_{u-s}$, we have

$$
\begin{gathered}
Y_{\Delta Q_{1}}=Y_{\Delta Q_{2}}=Y_{\Delta Q_{3}}=\frac{1}{5} \operatorname{Tr} Y_{\tilde{\Delta}}, \\
Y_{\Delta u}=Y_{\Delta d}=\frac{1}{10} \operatorname{Tr} Y_{\tilde{\Delta}}, \quad(\mathrm{C} 27) \\
Y_{\Delta c}=Y_{\Delta t}=-\frac{1}{80}\left(7 \operatorname{Tr} Y_{\tilde{\Delta}}-30 \operatorname{Tr} Y_{\Delta E}+30 \sum_{i} q_{\phi_{i}}^{Y} Y_{\Delta \phi_{i}}\right), \\
Y_{\Delta s}=Y_{\Delta b}=\frac{1}{80}\left(23 \operatorname{Tr} Y_{\tilde{\Delta}}-30 \operatorname{Tr} Y_{\Delta E}+30 \sum_{i} q_{\phi_{i}}^{Y} Y_{\Delta \phi_{i}}\right) .
\end{gathered}
$$

Finally, for $T<T_{u-d}$, we have

$$
Y_{\Delta Q_{1}}=Y_{\Delta Q_{2}}=Y_{\Delta Q_{3}}=\frac{1}{5} \operatorname{Tr} Y_{\tilde{\Delta}},
$$

$$
\begin{aligned}
Y_{\Delta u} & =Y_{\Delta c}=Y_{\Delta t} \\
& =-\frac{1}{55}\left(2 \operatorname{Tr} Y_{\tilde{\Delta}}-15 \operatorname{Tr} Y_{\Delta E}+15 \sum_{i} q_{\phi_{i}}^{Y} Y_{\Delta \phi_{i}}\right), \\
Y_{\Delta d} & =Y_{\Delta s}=Y_{\Delta b} \\
& =\frac{1}{55}\left(13 \operatorname{Tr} Y_{\tilde{\Delta}}-15 \operatorname{Tr} Y_{\Delta E}+15 \sum_{i} q_{\phi_{i}}^{Y} Y_{\Delta \phi_{i}}\right) .
\end{aligned}
$$

where we take $\mu=2 \pi T$ and $\alpha_{2}\left(m_{Z}\right)=0.0337$ with $m_{Z}=91.2 \mathrm{GeV}$. Here, we ignore the milder RGE of the charged lepton Yukawa and fix it in the flavor basis to be $\hat{y}_{E}=\operatorname{diag}\left(2.8 \times 10^{-6}, 5.9 \times 10^{-4}, 1.0 \times 10^{-2}\right)$ (the result is independent of basis).

We set $M_{\text {ref }}=10^{12} \mathrm{GeV}$, and for initial conditions, we take $Y_{\Delta B}\left(z_{i}\right)=0$ with $z_{i}=10^{-3}$ and choose arbitrary values for $Y_{\Delta}\left(z_{i}\right) \sim 10^{-10}$ and $Y_{\Delta E}\left(z_{i}\right) \sim 10^{-10}$. The behavior of the curve is rather insensitive to a particular choice of $Y_{\Delta}\left(z_{i}\right)$ and $Y_{\Delta E}\left(z_{i}\right)$. Solving up to $z_{f}=10^{3}$, to an accuracy within percent level, one obtains the fitting function

$$
c_{B}(T)=1-e^{-\frac{T_{B}}{T}},
$$

where $T_{B}=2.3 \times 10^{12} \mathrm{GeV}$.

To estimate the transition temperatures related to quark Yukawa interactions, one needs to solve for $c_{H}(T)$ as

$$
c_{H}(T)=-\frac{Y_{\Delta H}(T)}{\operatorname{Tr} Y_{\tilde{\Delta}}(T)-2 \operatorname{Tr} Y_{\Delta E}(T)} .
$$

Here, $Y_{\Delta H}$ should be treated as an independent variable, and one will need to construct a Boltzmann equation for $Y_{\Delta H}$ taking account all the interactions that change the number of Higgs. 
[1] V. A. Kuzmin, V. A. Rubakov, and M. E. Shaposhnikov, On the anomalous electroweak baryon number nonconservation in the early universe, Phys. Lett. 155B, 36 (1985).

[2] C. S. Fong, Baryogenesis from symmetry principle, Phys. Lett. B 752, 247 (2016).

[3] C.S. Fong, Baryogenesis in the Standard Model and its supersymmetric extension, Phys. Rev. D 103, L051705 (2021).

[4] M. Fukugita and T. Yanagida, Baryogenesis without grand unification, Phys. Lett. B 174, 45 (1986).

[5] G. Sigl and G. Raffelt, General kinetic description of relativistic mixed neutrinos, Nucl. Phys. B406, 423 (1993).

[6] M. Beneke, B. Garbrecht, C. Fidler, M. Herranen, and P. Schwaller, Flavoured leptogenesis in the CTP formalism, Nucl. Phys. B843, 177 (2011).

[7] B. Garbrecht, F. Glowna, and P. Schwaller, Scattering rates for leptogenesis: Damping of lepton flavour coherence and production of singlet neutrinos, Nucl. Phys. B877, 1 (2013).

[8] P. S. Bhupal Dev, P. Millington, A. Pilaftsis, and D. Teresi, Kadanoff-Baym approach to flavour mixing and oscillations in resonant leptogenesis, Nucl. Phys. B891, 128 (2015).

[9] B. Garbrecht and P. Schwaller, Spectator effects during leptogenesis in the strong washout regime, J. Cosmol. Astropart. Phys. 10 (2014) 012.

[10] S. Blanchet, P. Di Bari, D. A. Jones, and L. Marzola, Leptogenesis with heavy neutrino flavours: From density matrix to Boltzmann equations, J. Cosmol. Astropart. Phys. 01 (2013) 041.

[11] W. Buchmuller and M. Plumacher, Spectator processes and baryogenesis, Phys. Lett. B 511, 74 (2001).

[12] E. Nardi, Y. Nir, J. Racker, and E. Roulet, On Higgs and sphaleron effects during the leptogenesis era, J. High Energy Phys. 01 (2006) 068.

[13] G. 't Hooft, Symmetry Breaking Through Bell-Jackiw Anomalies, Phys. Rev. Lett. 37, 8 (1976).

[14] M. D’Onofrio, K. Rummukainen, and A. Tranberg, Sphaleron Rate in the Minimal Standard Model, Phys. Rev. Lett. 113, 141602 (2014).

[15] G. D. Moore, Computing the strong sphaleron rate, Phys. Lett. B 412, 359 (1997).
[16] S. Davidson and S. Sarkar, Thermalization after inflation, J. High Energy Phys. 11 (2000) 012.

[17] K. Harigaya and K. Mukaida, Thermalization after/during Reheating, J. High Energy Phys. 05 (2014) 006.

[18] A. D. Sakharov, Violation of $C P$ Invariance, C asymmetry, and baryon asymmetry of the universe, Pis'ma Zh. Eksp. Teor. Fiz. 5, 32 (1967).

[19] L. Bento, Sphaleron relaxation temperatures, J. Cosmol. Astropart. Phys. 11 (2003) 002.

[20] J. M. Cline, K. Kainulainen, and K. A. Olive, Protecting the primordial baryon asymmetry from erasure by sphalerons, Phys. Rev. D 49, 6394 (1994).

[21] E. Nardi, Y. Nir, E. Roulet, and J. Racker, The importance of flavor in leptogenesis, J. High Energy Phys. 01 (2006) 164.

[22] S. Lavignac and B. Schmauch, Flavour always matters in scalar triplet leptogenesis, J. High Energy Phys. 05 (2015) 124.

[23] B. Garbrecht, P. Klose, and C. Tamarit, Relativistic and spectator effects in leptogenesis with heavy sterile neutrinos, J. High Energy Phys. 02 (2020) 117.

[24] A. Dueck and W. Rodejohann, Fits to SO(10) grand unified models, J. High Energy Phys. 09 (2013) 024.

[25] C. S. Fong, D. Meloni, A. Meroni, and E. Nardi, Leptogenesis in SO(10), J. High Energy Phys. 01 (2015) 111.

[26] T. Hambye, M. Raidal, and A. Strumia, Efficiency and maximal $C P$-asymmetry of scalar triplet leptogenesis, Phys. Lett. B 632, 667 (2006).

[27] D. J. Gross and F. Wilczek, Ultraviolet Behavior of Nonabelian Gauge Theories, Phys. Rev. Lett. 30, 1343 (1973).

[28] H. D. Politzer, Reliable Perturbative Results for Strong Interactions?, Phys. Rev. Lett. 30, 1346 (1973).

[29] I. Esteban, M. C. Gonzalez-Garcia, M. Maltoni, T. Schwetz, and A. Zhou, The fate of hints: updated global analysis of three-flavor neutrino oscillations, J. High Energy Phys. 09 (2020) 178.

[30] https://glx.sourceforge.io/.

[31] H. A. Weldon, Effective fermion masses of order gt in high temperature gauge theories with exact chiral invariance, Phys. Rev. D 26, 2789 (1982). 